\title{
Attitude Analysis and Robust Adaptive Backstepping Sliding Mode Control of Spacecrafts Orbiting Irregular Asteroids
}

\author{
Chunhui Liang ${ }^{1,2}$ and Yuanchun $\mathrm{Li}^{1,3}$ \\ ${ }^{1}$ College of Communication Engineering, Jilin University, Changchun 130012, China \\ ${ }^{2}$ College of Electrical and Information, Changchun Institute of Technology, Changchun 130012, China \\ ${ }^{3}$ Department of Control Engineering, Changchun University of Technology, Changchun 130012, China
}

Correspondence should be addressed to Yuanchun Li; liyc@mail.ccut.edu.cn

Received 8 December 2013; Revised 20 February 2014; Accepted 7 March 2014; Published 17 April 2014

Academic Editor: Xiaojie Su

Copyright (C) 2014 C. Liang and Y. Li. This is an open access article distributed under the Creative Commons Attribution License, which permits unrestricted use, distribution, and reproduction in any medium, provided the original work is properly cited.

\begin{abstract}
Attitude stability analysis and robust control algorithms for spacecrafts orbiting irregular asteroids are investigated in the presence of model uncertainties and external disturbances. Rigid spacecraft nonlinear attitude models are considered and detailed attitude stability analysis of spacecraft subjected to the gravity gradient torque in an irregular central gravity field is included in retrograde orbits and direct orbits using linearized system model. The robust adaptive backstepping sliding mode control laws are designed to make the attitude of the spacecrafts stabilized and responded accurately to the expectation in the presence of disturbances and parametric uncertainties. Numerical simulations are included to illustrate the spacecraft performance obtained using the proposed control laws.
\end{abstract}

\section{Introduction}

SMALL bodies including mainly asteroids and comets are studied by scientists because of the insight they can give into the history of the solar system. NASA missions are as follows: Galileo to Jupiter via asteroids Gaspra and Ida in 1989, Near Earth Asteroid Rendezvous (NEAR) Shoemaker to asteroid 433 Eros in 1996 [1, 2], and NASA Flyby Mission Deep Space 1 to asteroid Braille in 1998, Genesis-NASA Discovery Solar Wind Sample Return Mission in 2001. Hayabusa (Muses-C) is the Japan Aerospace Exploration Agency Sample Return Mission to Asteroid 25143 Itokawa [3, 4], and Rosetta is the ESA Comet Mission, flew by asteroids Steins and Lutetia [5].

While there is an increasing interest in such missions, the necessity and importance of orbital and attitude dynamics analyses of the small solar system bodies as the critical success factors of those missions are rising as well. The oblateness torque effects can be ignored for studying the attitude motion of spacecrafts around planetary bodies, while asteroids and comets usually have irregular shapes which lead to the complicated orbital and attitude dynamics in comparison with approximately spherical bodies such as the Earth. An asteroid's irregular shape, mass distribution, and the state of its rotation (rapid or slow) have significant effects on the evolution of spacecraft orbit and attitude motion. Scheeres and his coworkers have made a large number of contributions to the study of orbital motion about asteroids [6-9]. These effects especially may deteriorate the attitude performance significantly, which lead to unstable attitude motion and thereby failure of the space mission. Wang and $\mathrm{Xu}$ find that the attitude stability domain is modified significantly due to the significantly nonspherical shape and rapid rotation of the asteroid, and attitude stability subjected to the disturbance of the gravity gradient torque is generalized to a rigid spacecraft on a stationary orbit around an asteroid [10,11]. In order to solve this problem, it is important to understand the attitude motion of spacecrafts orbiting asteroids by deriving the stability conditions and thereupon develop effective control laws to neutralize the effects of asteroid shape and mass distributions. Riverin and Misra have proposed the attitude motion of the spacecraft depending heavily on the shape of the asteroid and the rotational state [12]. Then Misra and Panchenko have found the radius for which resonant pitch oscillations, considering the general three-dimensional attitude motion in 2006 [13]. Riverin and Misra have examined the spacecraft pitch motion assuming the spacecraft is in an equatorial orbit 


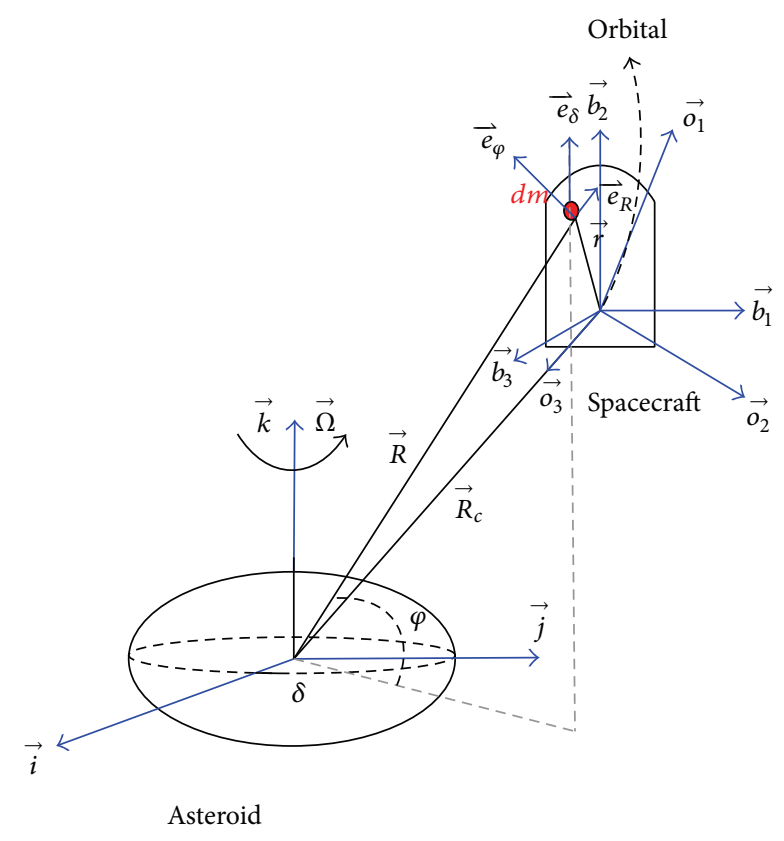

FIGURE 1: Coordinate Frames.

but spacecraft attitude control algorithms have not been perfectly investigated. Kumar and Shah have set up the general formulation of the spacecraft equations of motion in an equatorial eccentric orbit using Lagrangian method and made some analysis about the stability. Then the control laws for three-axis attitude control of spacecrafts have been developed and a closed-form solution of the system has been derived in [14]. Mahmut et al. have designed Lyapunov-based nonlinear feedback laws to control the rotational and translational motion of the spacecraft for an asteroid orbiting spacecraft in [15]. However, in the above articles about orbiting attitude motion, external perturbations acting on the spacecraft are not taken into account and the control laws are not robust.

Backstepping is a systematic and recursive design methodology for nonlinear feedback control. The idea is to select recursively some appropriate functions of state variables as pseudocontrol inputs for lower dimension subsystems of the overall system. Each backstepping stage results in a new pseudocontrol design, expressed in terms of the pseudocontrol designs from preceding design stages. When the procedure terminates, a feedback design for the true control input is the result which achieves the original design objective by virtue of a final Lyapunov function, which is formed by summing up the Lyapunov functions associated with each individual design stage. Sliding mode control is a nonlinear robust control and applicable to solve the tracking of nonlinear system $[16,17]$. The adaptive algorithm is adopted to estimate the external disturbances and uncertain parameters due to the highly complex environment in real time [18]. Moreover, owing to the robust control performance of adaptive backstepping control and sliding-mode control, many combined adaptive backstepping and sliding mode control schemes have appeared. Although good robust control strategies for uncertain nonlinear system and tracking problems have been proposed in $[19,20]$, adaptive backstepping sliding mode control is also effective and easier for implementation in real time. In this paper, the performance of spacecrafts orbiting irregular asteroids with perturbations is overall analysed, and attitude motion is influenced seriously. Moreover, the robust adaptive backstepping sliding mode control laws are proposed to compensate the uncertainties and perturbations and make the attitude angles decay and reach the null state, which ensure orbiting motion and space mission.

This paper is organized as follows. In Section 2, gravity gradient torque of spacecraft orbiting irregular asteroids is derived and three-dimensional attitude motion equations of the rigid spacecraft are first examined considering the perturbations, which is followed by deriving the linearized system model. In Section 3, the stability analysis about the spacecraft is presented in retrograde orbits and direct orbits with the orbital radius. Then in Section 4, effective adaptive backstepping sliding mode control schemes for a spacecraft orbiting the asteroid Eros 433 are developed to stabilize the system. Computer simulations are carried out to illustrate the effectiveness of the control laws. Conclusions are presented in Section 5.

\section{System Equations of Motion}

2.1. Coordinate Frames. At first, the following Coordinate Frames are set up to make the problem clear, which are shown in Figure 1.

(1) Asteroid centered inertial frame $(\vec{I}, \vec{J}, \vec{K})$ : the origin of this frame is at the center of mass of the asteroid.

(2) Asteroid-fixed frame $(\vec{i}, \vec{j}, \vec{k})$ : the origin of this frame is at the center of mass of the asteroid, the vectors are aligned along the three centroidal principal axis of the smallest, the intermediate, and the largest moment of inertia, respectively. The asteroid rotational state relates the two frames, the unit vector $\vec{k}$ points in the same direction as $\vec{K}$. Asteroid-fixed frame $(\vec{i}, \vec{j}, \vec{k})$ is assumed to rotate with constant angular velocity $\vec{\Omega}=\Omega \cdot \vec{K}$.

(3) The spacecraft orbital frame $\left(\vec{o}_{1}, \vec{o}_{2}, \vec{o}_{3}\right)$ : the origin of this frame is at the center of mass of the spacecraft, $\vec{o}_{3}$ points towards the center of mass of the asteroid, $\vec{o}_{1}$ points towards the transverse direction in the orbital plane, and $\vec{o}_{2}$ satisfied the right hand rule. For equatorial orbits, the orbital frame is obtained from the inertial frame $(\vec{I}, \vec{J}, \vec{K})$ by a single rotation through an angle equal to the true anomaly $\eta$.

(4) The spacecraft-fixed frame $\left(\vec{b}_{1}, \vec{b}_{2}, \vec{b}_{3}\right)$ : aligned with the principal axes of the spacecraft, their orientation with respect to the orbital frame can be defined in terms of the attitude angles (roll, pitch, and yaw).

The sequence of rotations used here is yaw $(\lambda)$ around $\vec{b}_{1}$ axis, followed by pitch $(\theta)$ around $\vec{b}_{2}$ axis, and then followed by roll $(\gamma)$ around $\vec{b}_{3}$ axis. 
2.2. Attitude Kinematics Model. The following assumptions are made in deriving the equations of motion firstly.

(1) The spacecraft is rigid.

(2) The gravitational attraction of the asteroid is the main disturbance force acting on the spacecraft, and the solar radiation and solar gravitation are considered perturbation force.

(3) The rotation rate of the asteroid is constant.

(4) The orbital motion of the spacecraft is not affected by attitude dynamics.

(5) Moment of inertias is affected by the irregular gravitational force of small bodies.

(6) Orbital motion of the spacecraft is fully described as a closed, planar, and periodic orbit.

(7) The asteroid is assumed to be a rotating triaxial ellipsoid.

In view of the first assumption, the attitude motion can be described by Euler's equations of motion for a rigid body:

$$
\begin{gathered}
I_{1} \dot{\omega}_{1}-\left(I_{2}-I_{3}\right) \omega_{2} \omega_{3}=M_{y}+M_{1}+M_{\Delta 1}, \\
I_{2} \dot{\omega}_{2}-\left(I_{3}-I_{1}\right) \omega_{3} \omega_{1}=M_{p}+M_{2}+M_{\Delta 2}, \\
I_{3} \dot{\omega}_{3}-\left(I_{1}-I_{2}\right) \omega_{1} \omega_{2}=M_{r}+M_{3}+M_{\Delta 3}, \\
\dot{\lambda}=\omega_{b 1}+\tan \theta\left(\omega_{b 2} \sin \lambda+\omega_{b 3} \cos \lambda\right), \\
\dot{\theta}=\omega_{b 2} \cos \lambda-\omega_{b 3} \sin \lambda, \\
\dot{\gamma}=\frac{1}{\cos \theta}\left(\omega_{b 2} \sin \lambda+\omega_{b 3} \cos \lambda\right),
\end{gathered}
$$

where $I_{1}, I_{2}, I_{3}$ are the principal moments of inertia of the spacecraft, $\omega_{1}, \omega_{2}, \omega_{3}$ are the components of the angular velocity along the principal axes in the spacecraft-fixed frame, $\omega_{b 1}, \omega_{b 2}, \omega_{b 3}$ are the relative angular velocity of the spacecraft with respect to the orbital frame $\left(\vec{o}_{1}, \vec{o}_{2}, \vec{o}_{3}\right)$ expressed in the spacecraft-fixed frame, and $\omega_{b 1}, \omega_{b 2}, \omega_{b 3}$ can be calculated by the coordinate transformation matrix $M_{B O}$ from the orbital frame to the spacecraft-fixed frame:

$$
\begin{aligned}
& {\left[\begin{array}{lll}
\omega_{b 1} & \omega_{b 2} & \omega_{b 3}
\end{array}\right]^{T}=\left[\begin{array}{lll}
\omega_{1} & \omega_{2} & \omega_{3}
\end{array}\right]^{T}-M_{B O}\left[\begin{array}{lll}
0 & \dot{\eta} & 0
\end{array}\right]^{T} . } \\
& M_{B O}=\left[\begin{array}{ccc}
1 & 0 & 0 \\
0 & \cos \lambda & \sin \lambda \\
0 & -\sin \lambda & \cos \lambda
\end{array}\right]\left[\begin{array}{ccc}
\cos \theta & 0 & -\sin \theta \\
0 & 1 & 0 \\
\sin \theta & 0 & \cos \theta
\end{array}\right] \\
& \times\left[\begin{array}{ccc}
\cos \gamma & \sin \gamma & 0 \\
-\sin \gamma & \cos \gamma & 0 \\
0 & 0 & 1
\end{array}\right] .
\end{aligned}
$$

And $M_{y}, M_{p}$, and $M_{r}$ are the components of the external control moment, $M_{1}, M_{2}, M_{3}$ are the components of the gravitational field of the asteroid, $M_{\Delta 1}, M_{\Delta 2}, M_{\Delta 3}$ are the components of the perturbation force, and $\dot{\eta}$ is the instantaneous orbital rate. Therefore, the full nonlinear equations of the attitude motion have been obtained by (1a), (1b), (2a), and (2b).
In view of the fifth assumption, the gravitational field of the asteroid is the primary and complex effect term which needs to be discussed in detail.

2.3. Gravity Gradient Torque. The gravitational potential of any arbitrary primary can be written in spherical harmonic series $[21,22]$ :

$$
\begin{array}{r}
U=\frac{G M}{R}\left\{1+\sum_{l=2}^{\infty}\left[C_{n 0}\left(\frac{R_{e}}{R}\right)^{l} P_{l}(\sin \varphi)\right.\right. \\
+\sum_{m=1}^{l}\left(\left(\frac{R_{e}}{R}\right)^{l} P_{l m}(\sin \varphi)\right. \\
\times\left(C_{l m} \cos m \delta\right. \\
\left.\left.\left.\left.+S_{l m} \sin m \delta\right)\right)\right]\right\},
\end{array}
$$

where $R$ is the distance of an orbiting particle from the center of mass of the primary and $R_{e}$ is the characteristic length of the primary, while $\varphi$ and $\delta$ are, respectively, the latitude and longitude of the orbiting particle measured in an asteroidfixed frame. The terms $P_{l}(\sin \varphi)$ are Legendre polynomials of degree $l$ and order 0 , and terms $P_{l m}(\sin \varphi)$ are associated Legendre polynomials of degree $l$ and order $m$. The two kinds of terms are given as

$$
\begin{gathered}
P_{l}(\sin \varphi)=\frac{1}{2^{l} l} \frac{d^{l}}{d(\sin \varphi)^{l}}\left\{\left[(\sin \varphi)^{2}-1\right]^{l}\right\} \\
P_{l m}(\sin \varphi)=\left[(\sin \varphi)^{2}-1\right]^{m / 2} \frac{d^{m} P_{l}(\sin \varphi)}{d(\sin \varphi)^{m}}
\end{gathered}
$$

The corresponding $C_{l m}$ and $S_{l m}$ are known as harmonic coefficients. When $m=l \neq 0$, they are called sectorial harmonic coefficients, and $P_{l m}(\sin \varphi)=P_{l}(\sin \varphi)$; the corresponding $C_{l 0}$ are known as zonal harmonic coefficients of order 0 . The coefficients $C_{l 0}$ specify the oblateness of the asteroid while $C_{l m}$ characterize the ellipticity of the asteroid's equator. For the Earth, $C_{20}$ is $O\left(10^{-3}\right)$ and the other coefficients are $O\left(10^{-6}\right)$. However, for some familiar asteroids these coefficients can be as high as $O\left(10^{-2}\right)$. Thus, the irregular shape of an asteroid can have a much stronger effect on attitude dynamics. We approximate the small body is a homogeneous triaxial ellipsoid with axes $a, b$, and $c$ in order to simplify the problem. We can calculate the coefficients as follows.

$S_{l m}=0$ for all $l$ or $m, C_{l m}=0$ for $l$ or $m$ are odd and while other conditions

$$
\begin{aligned}
C_{l m}= & \frac{3}{R_{e}^{l}} \frac{(l / 2) !(l-m) !}{2^{m}(l+3)(l+1) !}\left(2-\delta_{0 m}\right) \\
& \times \sum_{i=0}^{\operatorname{int}((l-m) / 4)}\left(\left(a^{2}-b^{2}\right)^{((m+4 i) / 2)}\right.
\end{aligned}
$$




$$
\begin{aligned}
& \left.\times\left[c^{2}-\left(\frac{1}{2}\right)\left(a^{2}+b^{2}\right)\right]^{((l-m-4 i) / 2)}\right) \\
& \times\left(16^{i}\left(\frac{l-m-4 i}{2}\right) !\left(\frac{m+2 i}{2}\right) ! i !\right)^{-1}
\end{aligned}
$$

$\delta_{0 m}$ is Kronecker symbol, and the value is

$$
\delta_{0 m}= \begin{cases}0, & m=0 \\ 1, & m=1\end{cases}
$$

For our purposes we have stopped the expansion of (3) to the second order, so we get the following coefficient:

$$
C_{20}=\frac{2 c^{2}-\left(a^{2}-b^{2}\right)}{10 R_{0}^{2}}, \quad C_{22}=\frac{a^{2}-b^{2}}{20 R_{0}^{2}} .
$$

The gravitational force acting on a particle of mass $d m$ at a distance $R$ from the asteroid center of mass, having latitude $\varphi$ and longitude $\delta$, is given by

$$
\overrightarrow{d F}=\left[\frac{\partial U}{\partial R} \vec{e}_{R}+\frac{1}{R \cos \varphi} \frac{\partial U}{\partial \delta} \vec{e}_{\delta}+\frac{1}{2} \frac{\partial U}{\partial \varphi} \vec{e}_{\varphi}\right] d m,
$$

where $U$ is given in (3), while $\vec{e}_{R}, \vec{e}_{\varphi}, \vec{e}_{\delta}$ are unit vectors associated with the spherical coordinate system $R, \varphi, \delta$ as shown in Figure 1 . The position vector $R$ of the element can be expressed as

$$
\vec{R}=\vec{R}_{c}+\vec{r}
$$

where $\vec{R}_{c}$ is the position vector of the center of mass of the spacecraft relative to the asteroid center of mass, while $\vec{r}$ is the position vector of the element in the spacecraft frame. We assume that $R$ and $R_{c}$ are much greater than $r$. Clearly

$$
R=\left|\vec{R}_{c}+\vec{r}\right|, \quad \vec{e}_{R}=\frac{\vec{R}_{c}+\vec{r}}{\left|\vec{R}_{c}+\vec{r}\right|}, \quad \vec{e}_{\delta}=\vec{e}_{R} \times \vec{e}_{\varphi}
$$

In conclusion, the gravity gradient torque on the spacecraft can then be determined from

$$
\begin{gathered}
\overrightarrow{d F}=d \vec{F}_{R}+\overrightarrow{d F}_{\delta}+d \overrightarrow{F F}_{\varphi}, \\
M_{g}=\int r \times d f .
\end{gathered}
$$

Evaluation of this torque involves expansion of the various powers of $\left|\vec{R}_{c}+\vec{r}\right|$ using the binomial theorem and neglecting terms involving third and higher powers of $|r| /\left|R_{c}\right|$.

Let $M_{g}=\left[\begin{array}{lll}M_{1} & M_{2} & M_{3}\end{array}\right]$ denote the gravity gradient torque in the spacecraft-fixed frame $\left(\vec{b}_{1}, \vec{b}_{2}, \vec{b}_{3}\right)$ and let $I$ denote the inertia matrix for the spacecraft, which is given as

$$
I=\left[\begin{array}{lll}
I_{1} & & \\
& I_{2} & \\
& & I_{3}
\end{array}\right]
$$

The unit vectors $\vec{e}_{R}, \vec{e}_{\varphi}, \vec{e}_{\delta}$ appearing in (8), (10), and (11) can now be expressed in terms of the yaw, pitch, and roll. The gravity-gradient torque components $M_{i}(i=1,2,3)$ in the spacecraft-fixed frame $\left(\vec{b}_{1}, \vec{b}_{2}, \vec{b}_{3}\right)$ can be written as follows after some algebra:

$$
\begin{aligned}
M_{1}=\frac{G M}{R_{c}^{3}}\left[(3+5 \alpha)\left(I_{3}-I_{2}\right) \cos \lambda \cos ^{2} \theta \sin \lambda\right. \\
+5 \beta\left(-\frac{2}{5} I_{1} \cos \lambda \sin \gamma\right. \\
\left.\left.+\left(I_{1}-I_{2}+I_{3}\right) \sin \lambda \cos ^{2} \theta \cos \gamma\right)\right],
\end{aligned}
$$

$$
\begin{aligned}
M_{2}=\frac{G M}{R_{c}^{3}}[(3+5 \alpha) & \left(I_{3}-I_{1}\right) \cos \lambda \cos \theta \sin \theta \\
+5 \beta(- & \frac{2}{5} I_{2}(\sin \lambda \sin \theta \sin \gamma-\cos \lambda \cos \gamma) \\
& +\left(I_{2}-I_{1}+I_{3}\right) \\
& \times(\sin \lambda \sin \theta \sin \gamma \\
& \left.+\sin ^{2} \theta \cos \lambda \cos \gamma\right) \\
& \left.\left.+\left(I_{2}-I_{3}+I_{1}\right) \cos ^{2} \theta \cos \lambda \cos \gamma\right)\right],
\end{aligned}
$$

$$
\begin{gathered}
M_{3}=\frac{G M}{R_{c}^{3}}\left[(3+5 \alpha)\left(I_{1}-I_{2}\right) \sin \lambda \cos \theta \sin \theta\right. \\
+5 \beta\left(\frac{2}{5} I_{3}(\sin \lambda \cos \gamma-\cos \lambda \sin \theta \sin \gamma)\right. \\
+\left(I_{2}-I_{1}+I_{3}\right) \\
\times(\cos \lambda \sin \lambda \sin \gamma \\
\left.-\sin ^{2} \theta \sin \lambda \cos \gamma\right) \\
-\left(I_{1}-I_{2}+I_{3}\right) \\
\left.\left.\times \cos ^{2} \theta \sin \lambda \cos \gamma\right)\right]
\end{gathered}
$$

where $\alpha, \beta$ are defined as follows, respectively:

$$
\begin{gathered}
\alpha=\left[-\frac{3}{2} C_{20}+9 C_{22} \cos \left(2 \delta_{c}\right)\right]\left(\frac{R_{e}}{R_{c}}\right)^{2}, \\
\beta=\left[6 C_{22} \sin \left(2 \delta_{c}\right)\right]\left(\frac{R_{e}}{R_{c}}\right)^{2} .
\end{gathered}
$$


2.4. Three-Dimensional Motion for Equatorial Orbits. Threedimensional motion of a spacecraft in an equatorial orbit is considered, and the attitude motion is small. It is assumed that the asteroid is rotating with a constant angular velocity $\Omega \cdot \vec{K}$. Assuming the rotating orbit of the spacecraft is circular orbits, $\dot{\eta}=n$, where $n$ is constant and stands for the orbital angular velocity of the spacecraft. Therefore, the longitude of the center of mass of the spacecraft is then given by

$$
\delta_{c}=(n \pm \Omega) t,
$$

where the plus and minus signs apply for retrograde and direct orbits, respectively.

Furthermore, for small motion, the angular velocity components given in (1a), (1b), (2a), and (2b) become

$$
\left[\begin{array}{c}
\omega_{1} \\
\omega_{2} \\
\omega_{3}
\end{array}\right]=\left[\begin{array}{c}
\dot{\lambda}-\dot{\eta} \gamma \\
\dot{\theta}-\dot{\eta} \\
\dot{\gamma}+\dot{\eta} \lambda
\end{array}\right]
$$

Therefore, a set of linearized equations for small motion of spacecraft are obtained in (21)-(23) by introducing (14)(19) into (la) and (1b):

$$
\begin{gathered}
\ddot{\lambda}+\dot{\eta}\left(k_{1}-1\right) \dot{\gamma}+\left[\frac{G M}{R_{c}^{3}}(3+5 \alpha)+\dot{\eta}^{2}\right] k_{1} \lambda \\
-\left[\frac{1}{2} \frac{G M}{R_{c}^{3}} \beta\left(3+5 k_{1}\right)+\ddot{\eta}^{2}\right] \gamma=u_{\lambda}+\Delta_{\lambda} \\
\ddot{\theta}-\ddot{\eta}+\frac{G M}{R_{c}^{3}}(3+5 \alpha) k_{2} \theta-\frac{1}{2} \frac{G M}{R_{c}^{3}} \beta\left(3+5 k_{2}\right)=u_{\theta}+\Delta_{\theta} \\
\ddot{\gamma}+\dot{\eta}\left(1-k_{3}\right) \dot{\lambda}+k_{3} \dot{\eta}^{2} \gamma \\
-\left[\ddot{\eta}+\frac{1}{2} \frac{G M}{R_{c}^{3}} \beta\left(3-5 k_{3}\right)\right] \lambda=u_{\gamma}+\Delta_{\gamma}
\end{gathered}
$$

where $k_{1}=\left(I_{2}-I_{3}\right) / I_{1}, k_{2}=\left(I_{1}-I_{3}\right) / I_{2}, k_{3}=\left(I_{2}-I_{1}\right) / I_{3}$, $u_{\lambda}, u_{\theta}, u_{\gamma}$ are control accelerations in three directions, and $\Delta_{\lambda}, \Delta_{\theta}, \Delta_{\gamma}$ are perturbation force accelerations consisting of gravitation higher order terms and solar radiation pressure, and so forth. Note that the pitch motion is decoupled from the roll and yaw motions, and this fact is similar to the case of a spacecraft orbiting symmetrically mass distributed planetary bodies.

\section{Analysis of Motion for Orbiting Circular Orbits}

3.1. Regular Resonance Analysis and Numerical Results of Pitch Motion. For circular orbits, $\dot{\eta}=n, \ddot{\eta}=0, G M / R_{c}^{3}=n^{2}$. For the understanding of the pitch behavior, let us consider small motion. Equation (22) then reduces to

$$
\ddot{\theta}+n^{2}(3+5 \alpha) k_{2} \theta-\frac{1}{2} n^{2} \beta\left(3+5 k_{2}\right)=0
$$

One can cast (24) which represents a harmonically excited system with periodic stiffness. If $k_{2}$ is negative, the pitch
TABLE 1: Simulation parameters.

\begin{tabular}{lc}
\hline Simulation parameters & Simulation value \\
\hline $\begin{array}{l}\text { Characteristic length of the asteroid } R_{e} \\
(\mathrm{~km})\end{array}$ & 9.933 \\
\hline $\begin{array}{l}\text { Harmonic coefficients } C_{20} \\
\text { Harmonic coefficients } C_{22}\end{array}$ & -0.0878 \\
$\begin{array}{l}\text { Spacecraft mass distribution } \\
\text { parameters } k_{1}, k_{2}, k_{3}\end{array}$ & 0.0439 \\
$\begin{array}{l}\text { Asteroid's gravitational constant } \\
\text { parameter } \mu\left(\mathrm{km}^{3} / \mathrm{s}^{2}\right)\end{array}$ & $1 / 3,1 / 3,1 / 3$ \\
Asteroid's spin rate $\Omega(\mathrm{rad} / \mathrm{s})$ & 876171 \\
\end{tabular}

motion is normally unstable; hence, the case of positive is considered in this paper.

Since $k_{2}<1,\left|C_{20}\right|<0.1$, choosing the minus sign in (19), parametric resonance occurs when the spacecraft is in a retrograde orbit when the asteroid and orbital angular velocities are related [14] approximately by

$$
R_{c}=\left(\frac{G M}{\Omega^{2}}\right)^{1 / 3}\left[\frac{j \mp\left(3 k_{2}\right)^{1 / 2}}{j}\right]^{2 / 3}, \quad j=1,2,3, \ldots
$$

Regular resonance takes place when $j=2$; that is,

$$
R_{c}=\left(\frac{G M}{\Omega^{2}}\right)^{1 / 3}\left[1 \mp \frac{\sqrt{3 k_{2}}}{2}\right]^{2 / 3}
$$

3.2. Results for the Three-Dimensional Case. Equations (21)(23) are quite complex and must be solved numerically with the initial conditions of roll, pitch, and yaw $\lambda(0)=0.1 \mathrm{rad}$, $\dot{\lambda}(0)=0 ; \theta(0)=0.1 \mathrm{rad}, \dot{\theta}(0)=0 ; \gamma(0)=0.1 \mathrm{rad}, \dot{\gamma}(0)=0$.

Table 1 gives simulation parameters for three-dimensional motion about Eros 433. Figures 2, 3, 4, 5, 6, 7, 8, 9, and 10 give the three-dimensional motions of a spacecraft orbiting Eros in equatorial circular retrograde orbits at $R_{c}=48 \mathrm{~km}, 31 \mathrm{~km}, 15 \mathrm{~km}$, respectively, without taking into account perturbation torque.

The pitch motion is quite regular with amplitude of $0.1 \mathrm{rad}$ at $R_{c}=48 \mathrm{~km}$ with the above initial conditions, and amplitudes of the roll and yaw motions are all steady. When orbital radius is decrease the three-dimensional motions especially pitch are irregular, and the irregularity is becoming apparent when the spacecraft is nearer to the asteroid. Similarly Figures $11,12,13,14,15,16,17,18,19,20,21$, and 22 give the three-dimensional motions of a spacecraft orbiting Eros in equatorial circular direct orbits at $R_{c}=50 \mathrm{~km}, 35 \mathrm{~km}, 27 \mathrm{~km}$, $26 \mathrm{~km}$, respectively, without taking into account perturbation torques. The three-dimensional motion has the same trend with retrograde orbits when the orbital radius are decrease, but the roll and raw motions become instable when $R_{c}=$ $26 \mathrm{~km}$.

It is observed that irregularities of attitude angles are more obvious when the spacecraft is nearer to the small body, which make the vibration amplitude and frequency of the spacecrafts more strong. We can draw the conclusion from the simulation results that the irregular gravity-gradient 


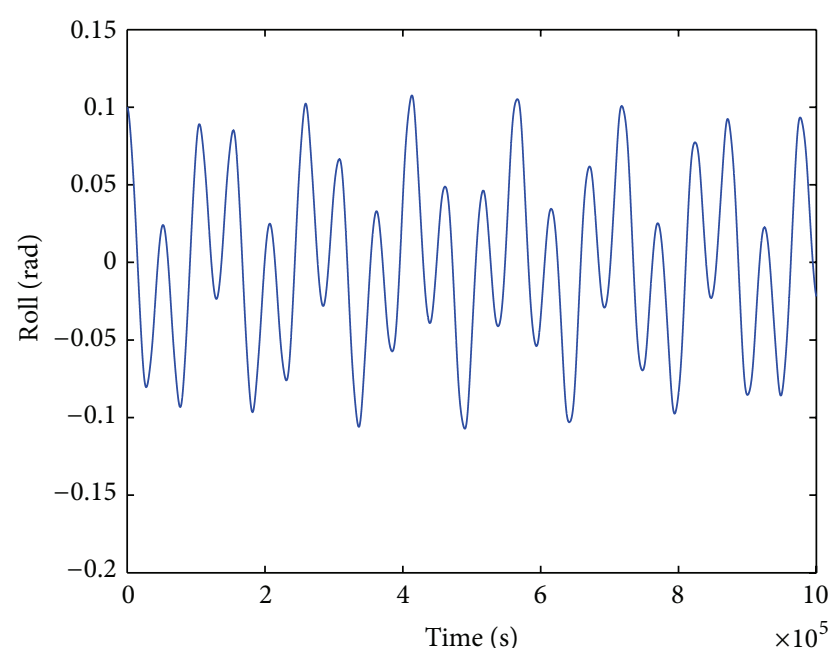

FIGURE 2: Roll $R_{c}=48 \mathrm{~km}, k_{2}=1 / 3$.

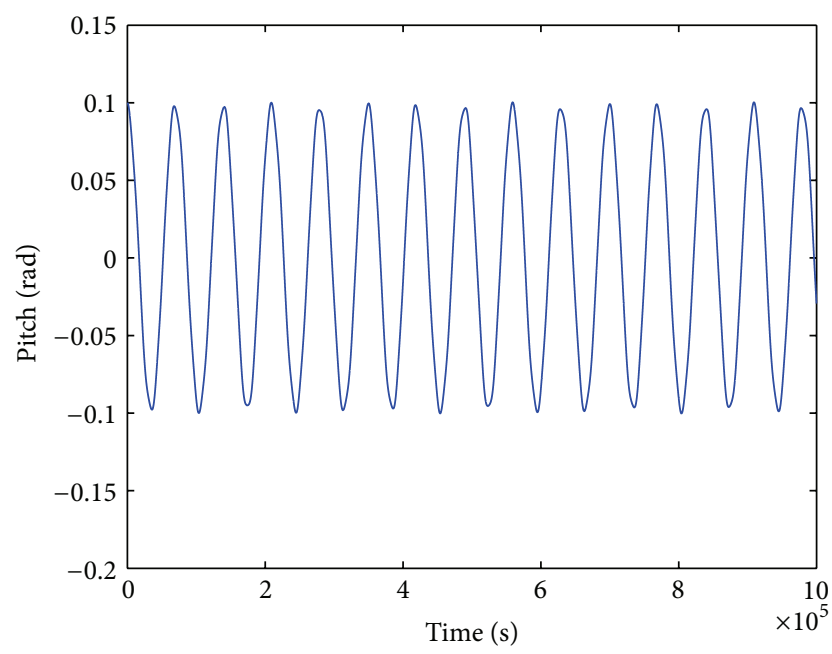

FIgURE 3: Pitch $R_{c}=48 \mathrm{~km}, k_{2}=1 / 3$.

torque of the asteroid has the primary and complex effect on the spacecraft orbiting motion. The spacecraft may get out of the orbit if external perturbations such as solar radiation pressure are taken into account. So it is essential to design the robust control algorithms to compensate the uncertainties and perturbations and stabilize the attitude angles.

\section{Controller Design}

In this section, we present adaptive sliding mode control laws based on backstepping which achieves three-axes stabilized nadir-pointing attitude. In other words, the control objective is to align the spacecraft-fixed axes with the orbital reference axes. The desired attitude angles yaw $(\lambda)$, pitch $(\theta)$, and roll $(\gamma)$ are zero.

4.1. Backstepping Control. The basic idea of backstepping method is decomposition of a complicated nonlinear system, then designing Lyapunov function and suppositional control

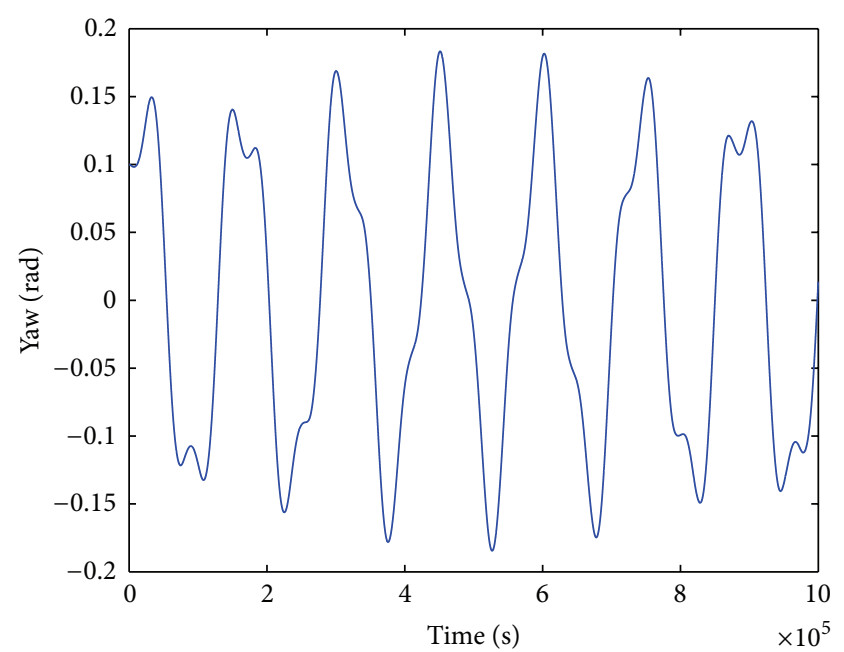

Figure 4: Yaw $R_{c}=48 \mathrm{~km}, k_{2}=1 / 3$.

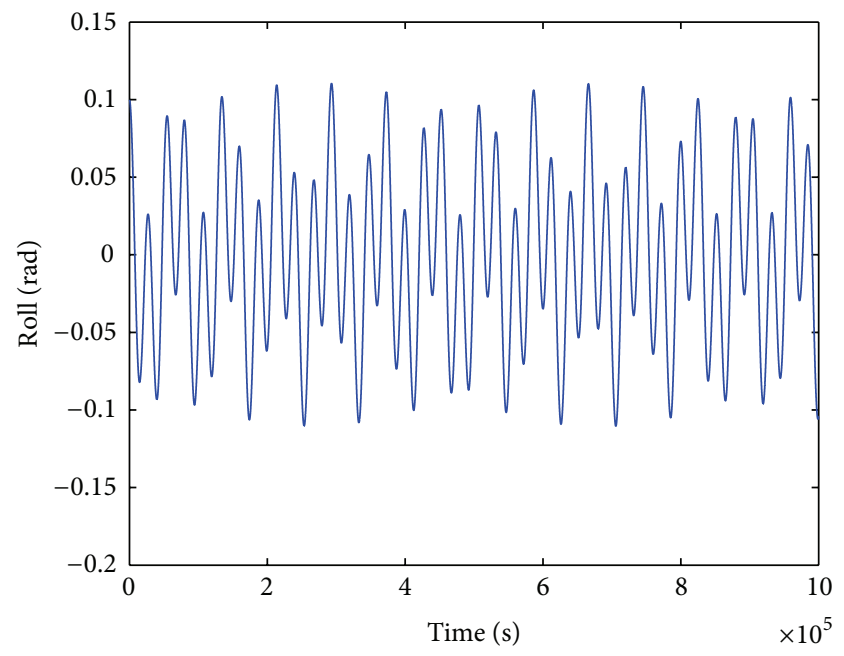

FIGURE 5: Roll $R_{c}=31 \mathrm{~km}, k_{2}=1 / 3$.

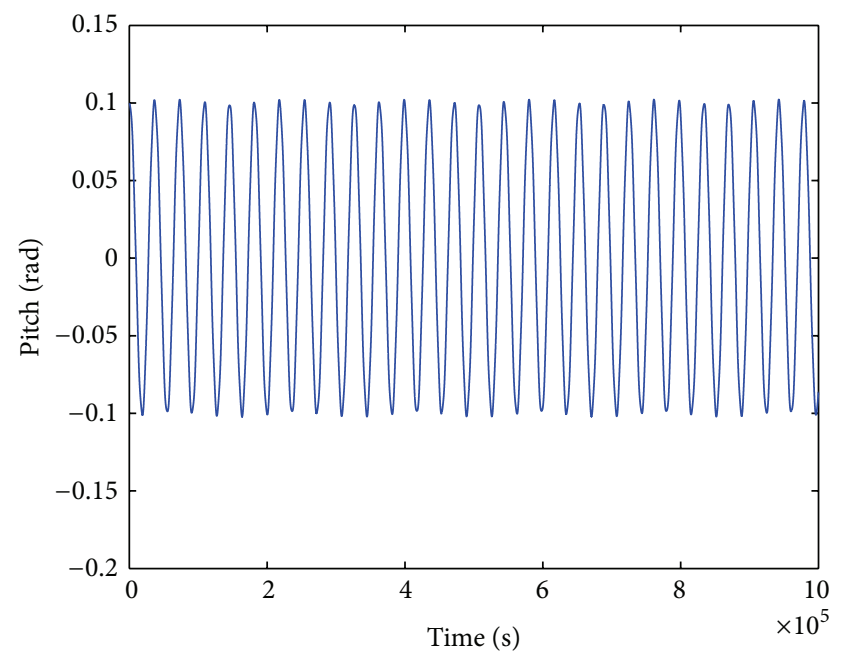

Figure 6: Pitch $R_{c}=31 \mathrm{~km}, k_{2}=1 / 3$. 


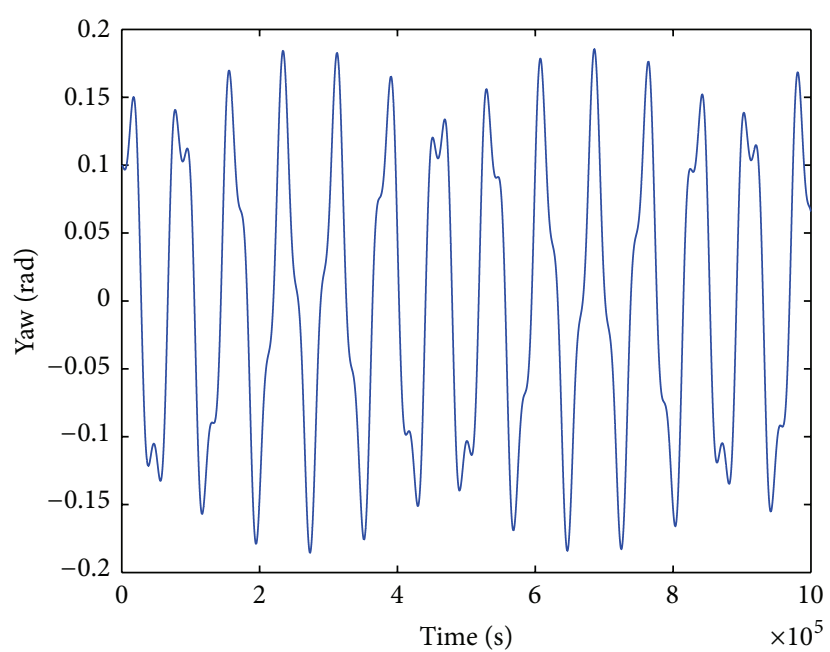

Figure 7: Yaw $R_{c}=31 \mathrm{~km}, k_{2}=1 / 3$.

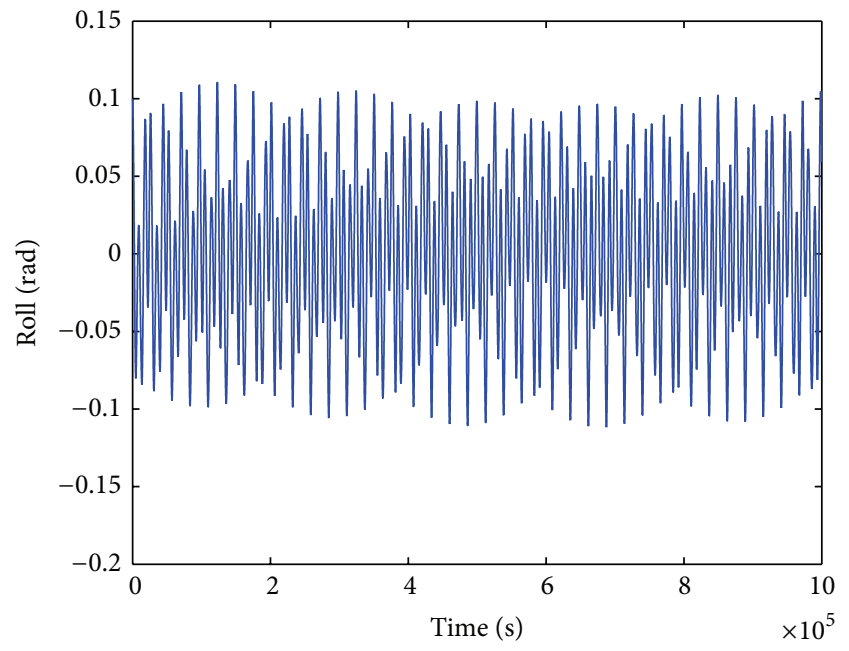

Figure 8: Roll $R_{c}=15 \mathrm{~km}, k_{2}=1 / 3$.

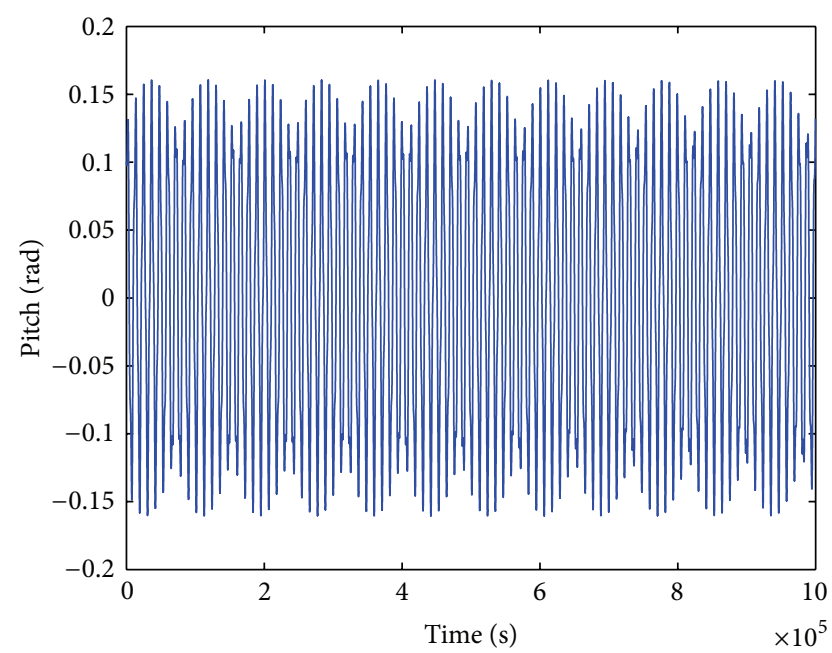

FIgURE 9: Pitch $R_{c}=15 \mathrm{~km}, k_{2}=1 / 3$.

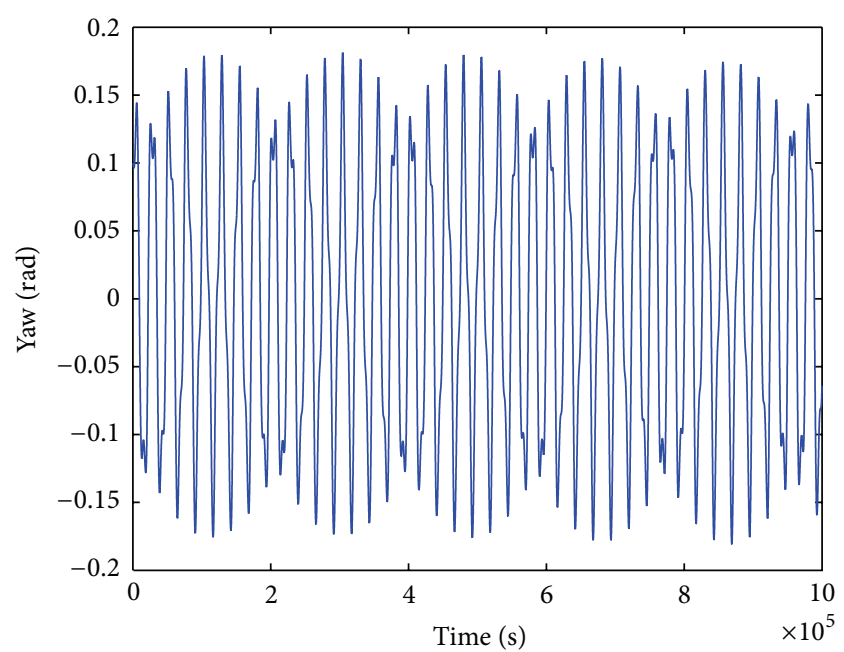

Figure 10: Yaw $R_{c}=15 \mathrm{~km}, k_{2}=1 / 3$.

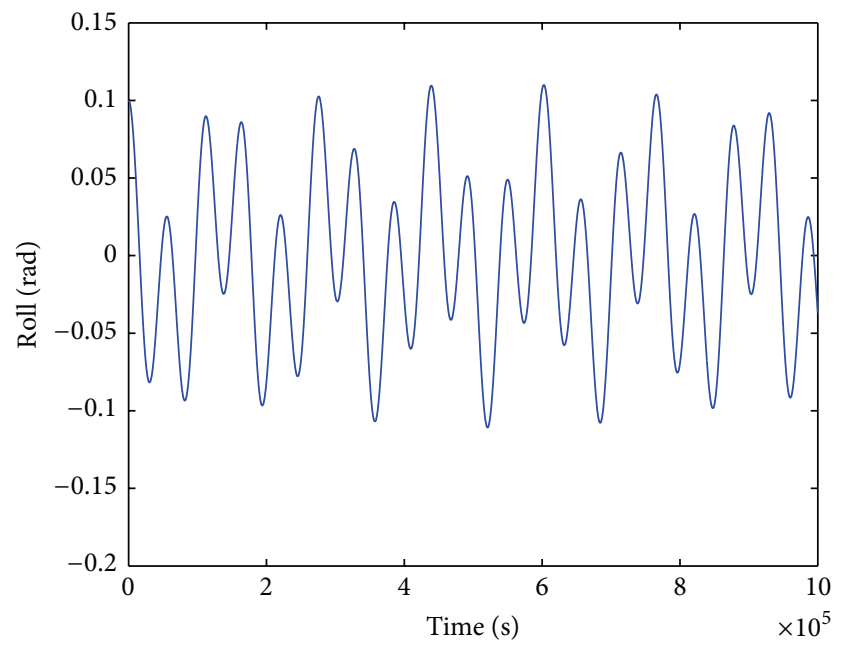

FIGURE 11: Roll $R_{c}=50 \mathrm{~km}, k_{2}=1 / 3$.

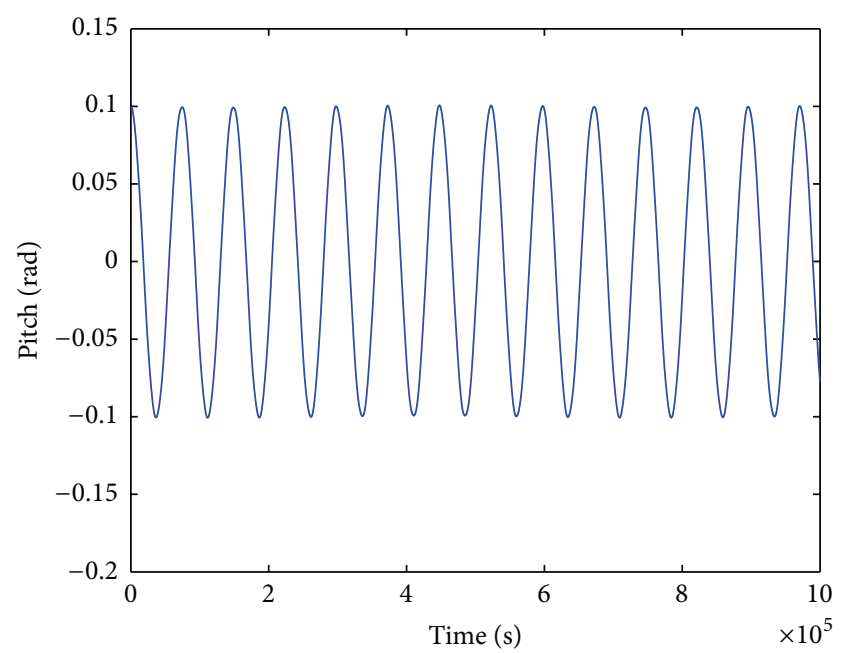

Figure 12: Pitch $R_{c}=50 \mathrm{~km}, k_{2}=1 / 3$. 


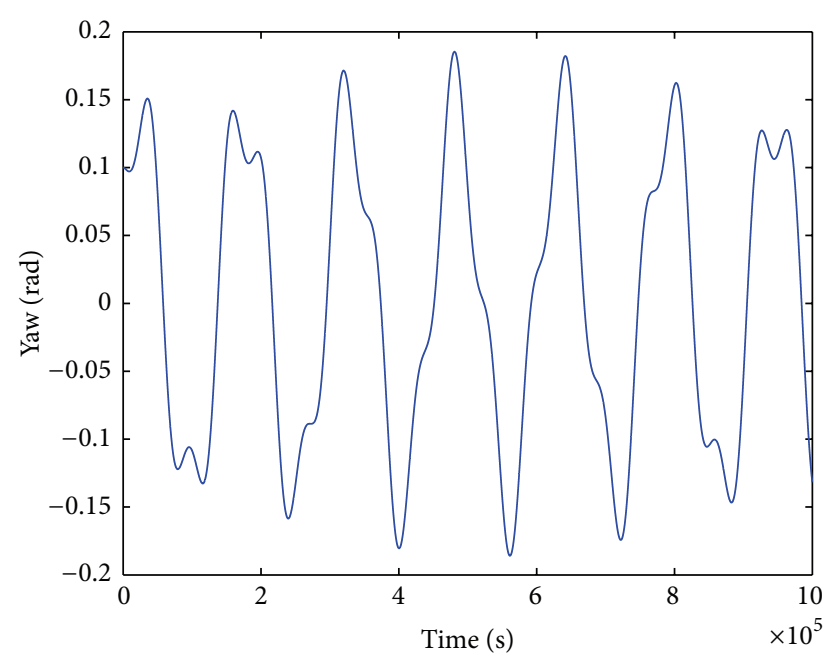

Figure 13: Yaw $R_{c}=50 \mathrm{~km}, k_{2}=1 / 3$.

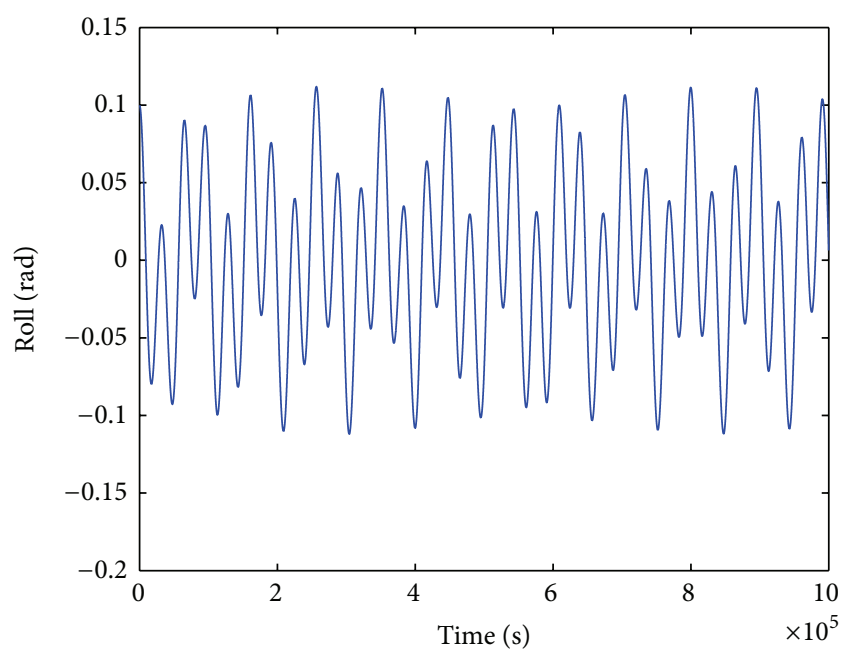

FIgURE 14: Roll $R_{c}=35 \mathrm{~km}, k_{2}=1 / 3$.

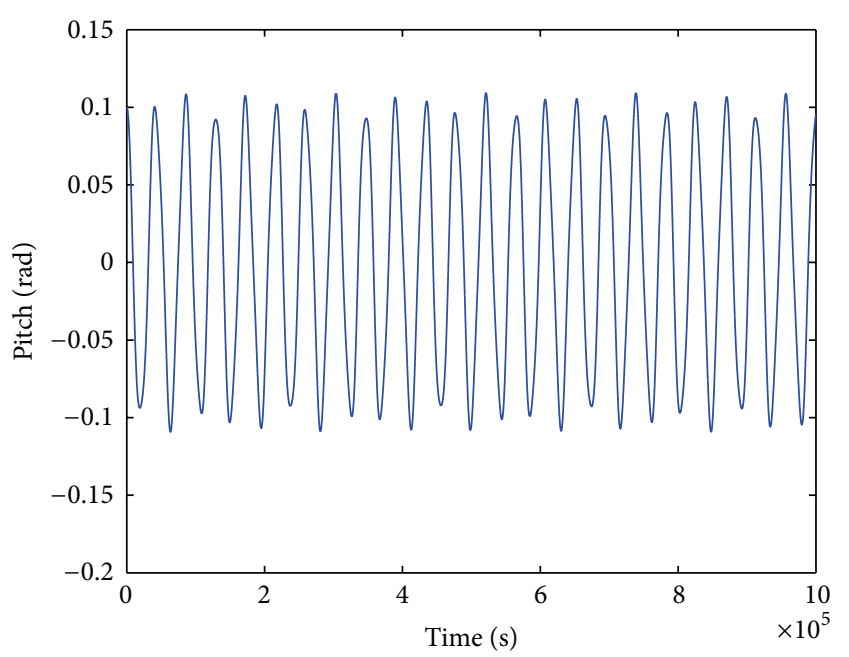

FIgURE 15: Pitch $R_{c}=35 \mathrm{~km}, k_{2}=1 / 3$.

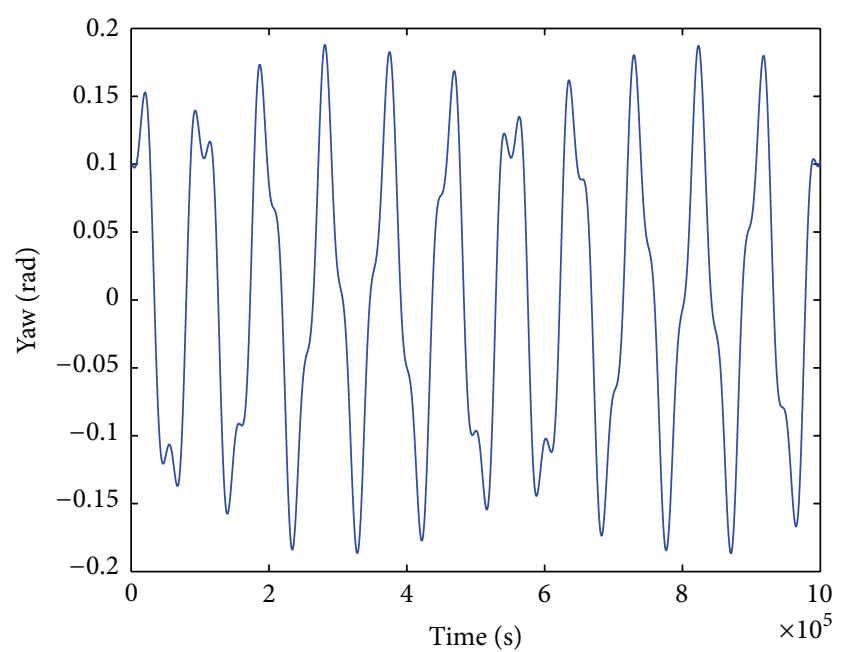

FIGURE 16: Yaw $R_{c}=35 \mathrm{~km}, k_{2}=1 / 3$.

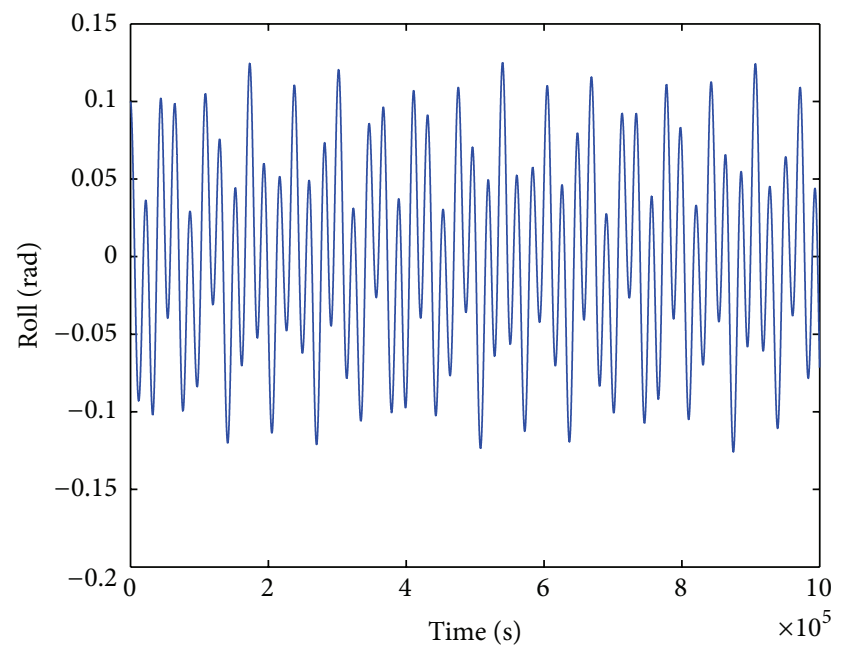

FIGURE 17: Roll $R_{c}=27 \mathrm{~km}, k_{2}=1 / 3$.

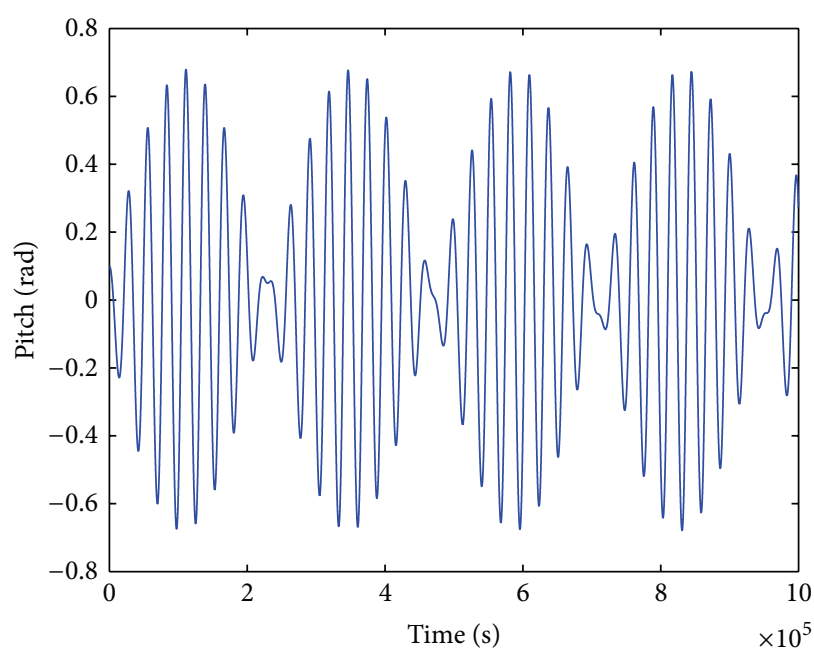

Figure 18: Pitch $R_{c}=27 \mathrm{~km}, k_{2}=1 / 3$. 


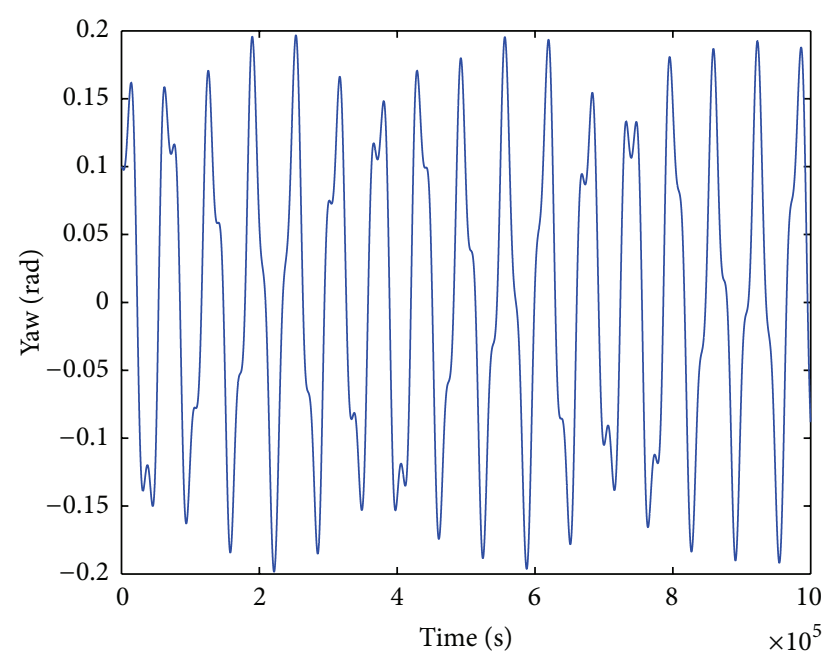

FIGURE 19: Raw $R_{c}=27 \mathrm{~km}, k_{2}=1 / 3$.

for the decomposed system. The final control laws are designed after backing to the overall system.

Regardless of perturbation, suppose (21), (22), and (23) are

$$
\begin{gathered}
\dot{x}_{1}=x_{2}, \quad \dot{x}_{2}=f\left(x_{1}, x_{2}\right)+b u \\
x_{1}=[\lambda, \theta, \gamma], \quad x_{2}=[\dot{\lambda}, \dot{\theta}, \dot{\gamma}] \\
f\left(x_{1}, x_{2}\right) \\
=\left[\dot{\eta}\left(k_{1}-1\right) \dot{\gamma}+\left[\frac{G M}{R_{c}^{3}}(3+5 \alpha)+\dot{\eta}^{2}\right] k_{1} \lambda\right. \\
-\left[\frac{1}{2} \frac{G M}{R_{c}^{3}} \beta\left(3+5 k_{1}\right)+\ddot{\eta}^{2}\right] \gamma-\ddot{\eta}+\frac{G M}{R_{c}^{3}}(3+5 \alpha) k_{2} \theta \\
-\frac{1}{2} \frac{G M}{R_{c}^{3}} \beta\left(3+5 k_{2}\right) \dot{\eta}\left(1-k_{3}\right) \dot{\lambda}+k_{3} \dot{\eta}^{2} \gamma \\
\left.-\left[\ddot{\eta}+\frac{1}{2} \frac{G M}{R_{c}^{3}} \beta\left(3-5 k_{3}\right)\right] \lambda\right], \quad b=\left[\begin{array}{l}
1 \\
1 \\
1
\end{array}\right] .
\end{gathered}
$$

Define position error $z_{1}=x_{1}-z_{d}$; $z_{d}$ is the expected trajectory $\dot{z}_{1}=\dot{x}_{1}-\dot{z}_{d}=x_{2}-\dot{z}_{d}$.

Assume the virtual control in

$$
\alpha_{1}=-c_{1} z_{1}+\dot{z}_{d} \quad\left(c_{1}>0\right) .
$$

Define $z_{2}=x_{2}-\alpha_{1}$ and Lyapunov function $V_{1}=(1 / 2) z_{1}^{2}$, so (29) is obtained:

$$
\dot{V}_{1}=z_{1} \dot{z}_{1}=z_{1}\left(x_{2}-\dot{z}_{d}\right)=z_{1}\left(z_{2}+\alpha_{1}-\dot{z}_{d}\right) .
$$

Introducing (28) into (29), $\dot{V}_{1}=-c_{1} z_{1}^{2}+z_{1} z_{2}$ is obtained. If $z_{2}=0$, then $\dot{V}_{1} \leq 0$.

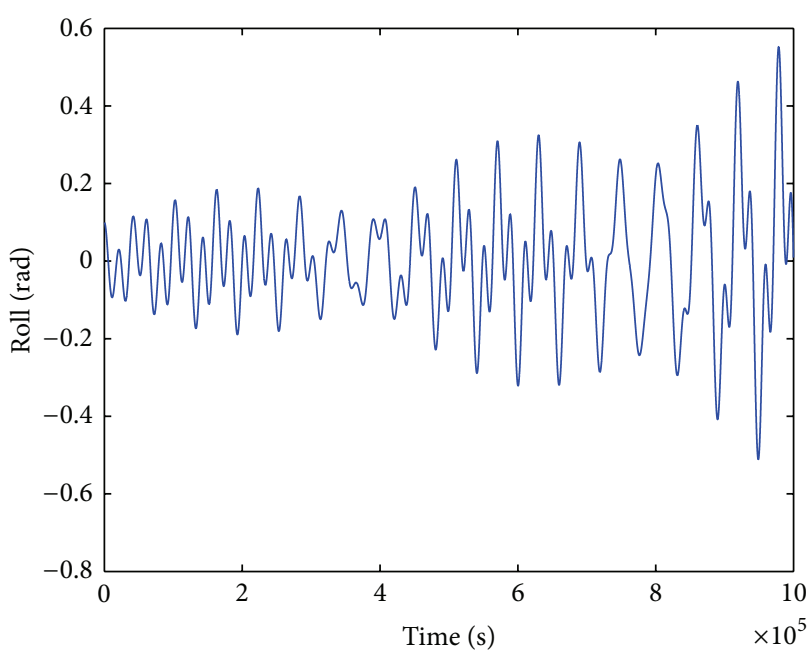

FIgURE 20: Roll $R_{c}=26 \mathrm{~km}, k_{2}=1 / 3$.

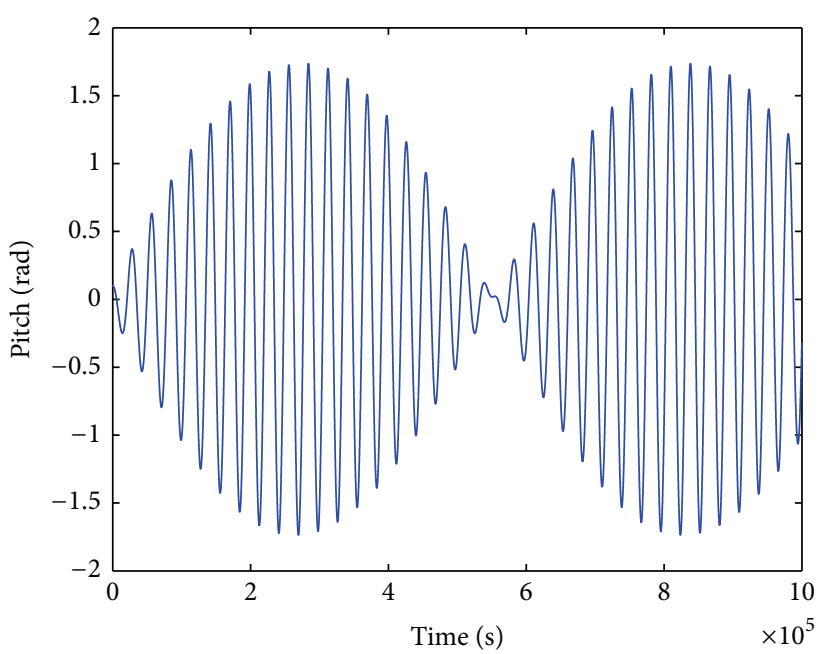

FIGURE 21: Pitch $R_{c}=26 \mathrm{~km}, k_{2}=1 / 3$.

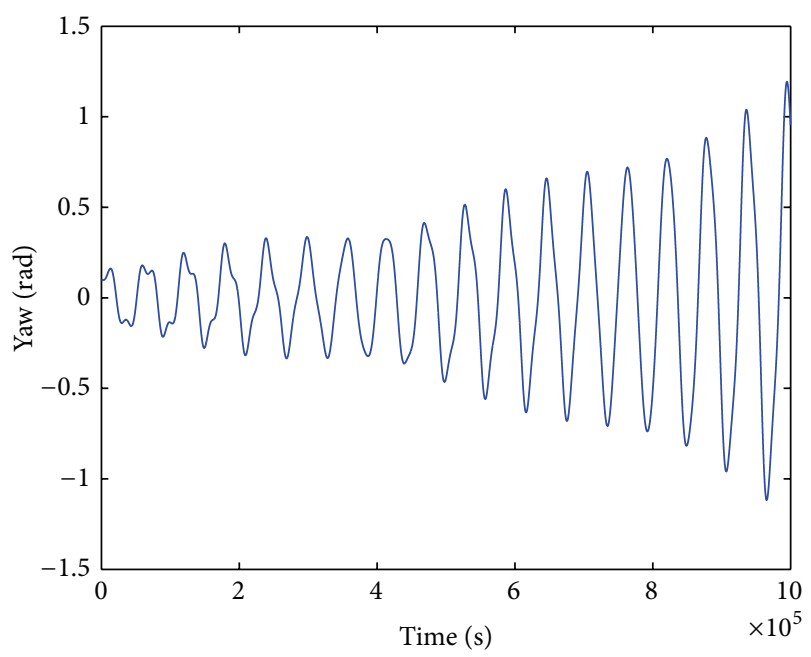

Figure 22: Pitch $R_{c}=26 \mathrm{~km}, k_{2}=1 / 3$. 


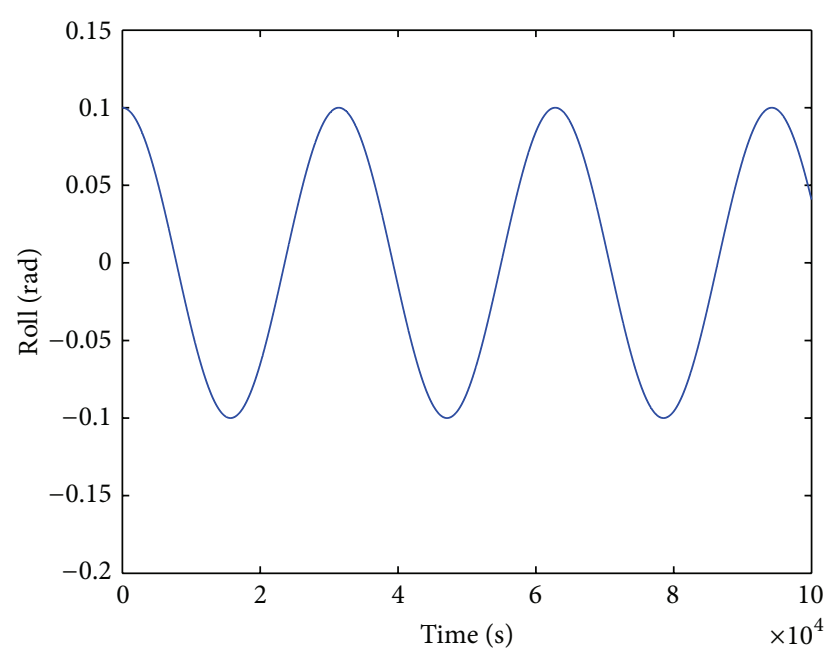

FIGURE 23: Roll response.

Define Lyapunov function $V_{2}=V_{1}+(1 / 2) z_{2}^{2}$; then

$$
\begin{aligned}
\dot{V}_{2} & =\dot{V}_{1}+z_{2} \dot{z}_{2} \\
& =-c_{1} z_{1}^{2}+z_{1} z_{2}+z_{2}\left[f\left(x_{1}, x_{2}\right)+b u+c_{1} \dot{z}_{1}-\ddot{z}_{d}\right] .
\end{aligned}
$$

The following control laws are obtained in

$u=\frac{1}{b}\left[-f\left(x_{1}, x_{2}\right)-c_{2} z_{2}-z_{1}-c_{1} \dot{z}_{1}+\ddot{z}_{d}\right]\left(c_{2}>0\right)$.

Thus, $\dot{V}_{2}=-c_{1} z_{1}^{2}-c_{2} z_{2}^{2} \leq 0$.

Spacecraft attitude angles pitch, roll, and yaw angles can reach regular resonance based on control law (31) with the initial conditions of roll, pitch, and yaw $\lambda(0)=0.1 \mathrm{rad}$, $\dot{\lambda}(0)=0 \mathrm{rad} ; \theta(0)=0.1 \mathrm{rad}, \dot{\theta}(0)=0 \mathrm{rad} ; \gamma(0)=0.1 \mathrm{rad}$, $\dot{\gamma}(0)=0$ rad. In the case of the simulation parameters described as Table 1, the desired attitude motion of the spacecraft can be specified by periodic functions with the desired amplitudes. The corresponding attitude motions are determined numerically in Figures 23, 24, and 25. Figures 26, 27 , and 28 give the control accelerations of three-dimensional motions. Backstepping control laws can make roll, pitch, and yaw track the desired periodic trajectory without external disturbances when the spacecraft in circular retrograde or direct orbits. The desired attitude angles of spacecraft are adopted periodic function as $z d 1=0.1 * \cos (0.0002 * t)$ in the experiments.

From the experimental results in Figures 23, 24, and 25 , backstepping control law (31) can guarantee the output signals stable, tracking the desired attitude of spacecraft globally and asymptotically without external perturbances.

4.2. Adaptive Backstepping Sliding Mode Control. With external disturbances and uncertain parameters, adaptive backstepping sliding mode control schemes are developed, which have been applied in uncertain systems [23]. It introduces the sliding mode control in backstepping design to modify the last step of backstepping algorithm and simplify the design of controller.

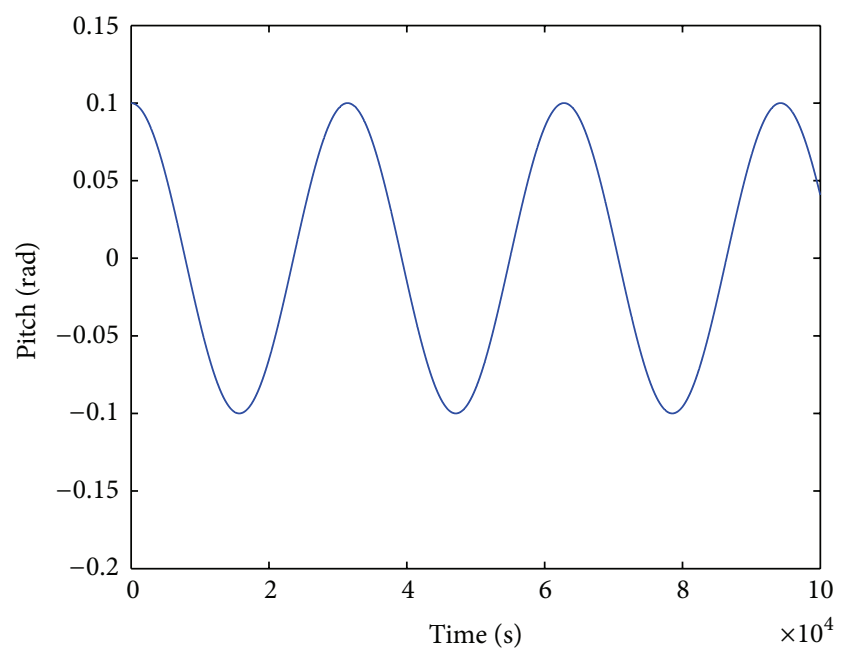

Figure 24: Pitch response.

Without loss of generality, suppose (21), (22), and (23) are

$$
\begin{gathered}
\dot{x}_{1}=x_{2}, \quad \dot{x}_{2}=f\left(x_{1}, x_{2}\right)+b u+F, \\
y=x_{1},
\end{gathered}
$$

$$
x_{1}=[\lambda, \theta, \gamma], \quad x_{2}=[\dot{\lambda}, \dot{\theta}, \dot{\gamma}],
$$

$$
\begin{aligned}
f\left(x_{1}, x_{2}\right) & \\
= & {\left[\dot{\eta}\left(k_{1}-1\right) \dot{\gamma}+\left[\frac{G M}{R_{c}^{3}}(3+5 \alpha)+\dot{\eta}^{2}\right] k_{1} \lambda\right.} \\
& -\left[\frac{1}{2} \frac{G M}{R_{c}^{3}} \beta\left(3+5 k_{1}\right)+\ddot{\eta}^{2}\right] \gamma-\ddot{\eta}+\frac{G M}{R_{c}^{3}}(3+5 \alpha) k_{2} \theta \\
& -\frac{1}{2} \frac{G M}{R_{c}^{3}} \beta\left(3+5 k_{2}\right) \dot{\eta}\left(1-k_{3}\right) \dot{\lambda}+k_{3} \dot{\eta}^{2} \gamma \\
& \left.-\left[\ddot{\eta}+\frac{1}{2} \frac{G M}{R_{c}^{3}} \beta\left(3-5 k_{3}\right)\right] \lambda\right], \quad b=\left[\begin{array}{l}
1 \\
1 \\
1
\end{array}\right] .
\end{aligned}
$$

$|F| \leq \bar{F}$ is the whole external disturbances and uncertain parameters, and we suppose it changes slowly; that is $\dot{F}=0$.

To begin with, define the position error $z_{1}=y-y_{d} ; y_{d}$ is the expected position:

$$
\dot{z}_{1}=\dot{y}-\dot{y}_{d}=x_{2}-\dot{y}_{d} .
$$

The stability term is $\alpha_{1}=c_{1} z_{1}$, and $c_{1}$ is positive constant.

Define Lyapunov function to be $V_{1}=(1 / 2) z_{1}^{2}$ and $z_{2}=$ $\dot{z}_{1}+\alpha_{1}=x_{2}-\dot{y}_{d}+\alpha_{1}$

$$
\dot{V}_{1}=z_{1} \dot{z}_{1}=z_{1}\left(x_{2}-\dot{y}_{d}\right)=z_{1}\left(z_{2}-\alpha_{1}\right)=z_{1} z_{2}-c_{1} z_{1}^{2} \text {. }
$$

Then, $\dot{z}_{2}=\dot{x}_{2}-\ddot{y}_{d}+\dot{\alpha}_{1}=f\left(x_{1}, x_{2}\right)+b u+F-\ddot{y}_{d}+\dot{\alpha}_{1}$.

Define Lyapunov function $V_{2}=V_{1}+(1 / 2) \sigma^{2} ; \sigma=k_{1} z_{1}+$ $z_{2}\left(k_{1}>0\right)$ is the switching function. 


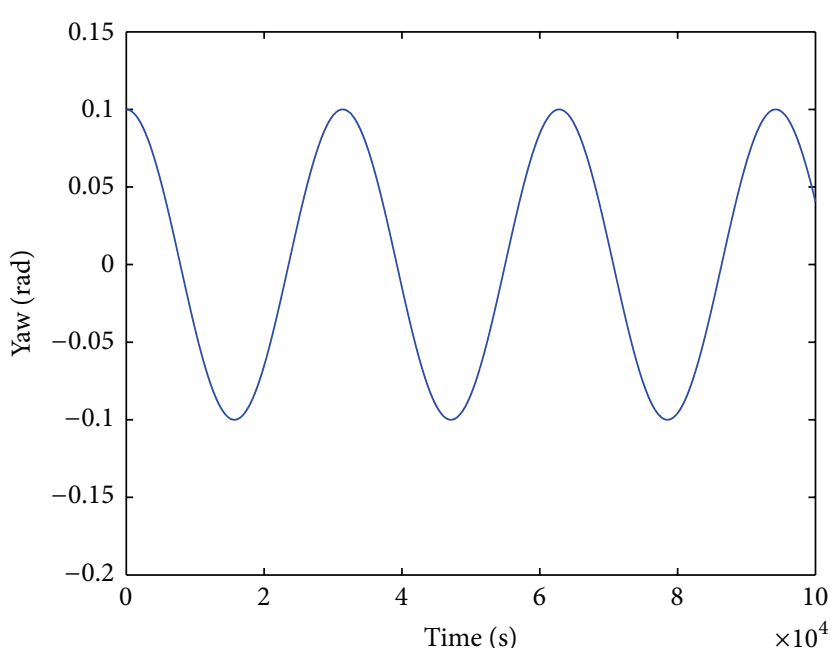

Figure 25: Yaw response.

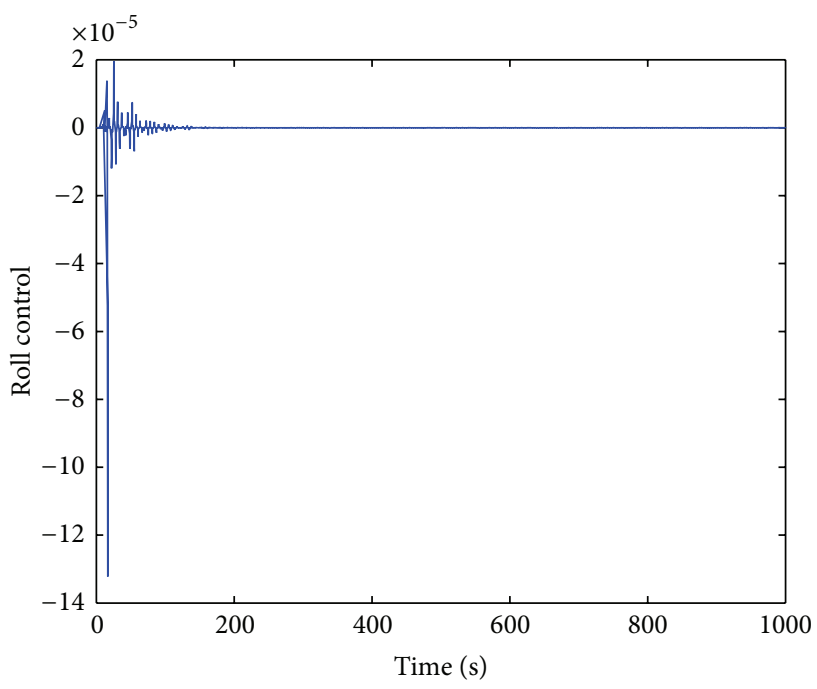

FiguRe 26: Roll control input.

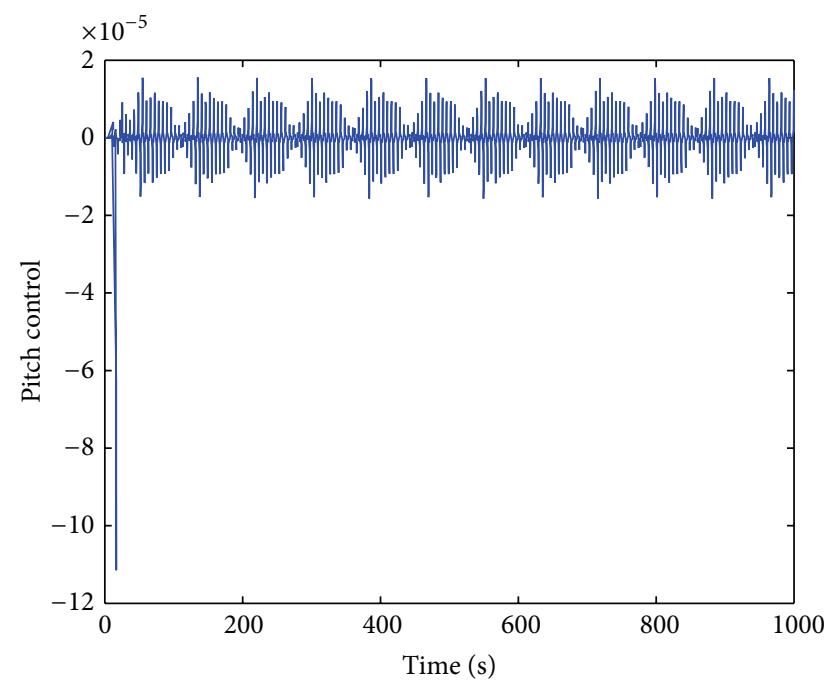

FIgURE 27: Pitch control input.

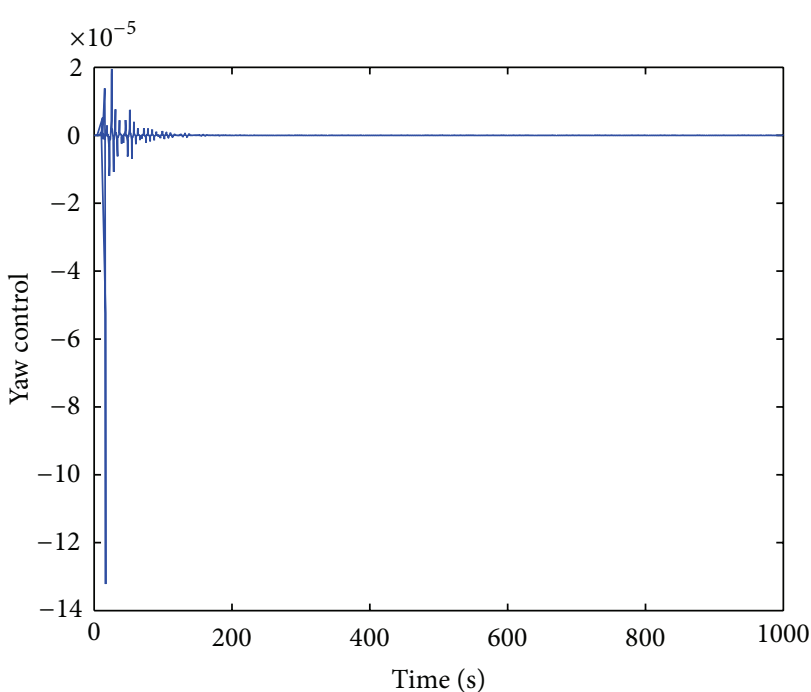

FIGURE 28: Yaw control input.

Taking the derivative of $V_{2}(35)$ is obtained:

$$
\begin{aligned}
\dot{V}_{2}= & \dot{V}_{1}+\sigma \dot{\sigma}=z_{1} z_{2}-c_{1} z_{1}^{2}+\sigma \dot{\sigma} \\
= & z_{1} z_{2}-c_{1} z_{1}^{2}+\sigma\left(k_{1} \dot{z}_{1}+\dot{z}_{2}\right) \\
= & z_{1} z_{2}-c_{1} z_{1}^{2} \\
& \quad+\sigma\left[k_{1}\left(z_{2}-c_{1} z_{1}\right)+f\left(x_{1}, x_{2}\right)+b u+F-\ddot{y}_{d}+\dot{\alpha}_{1}\right] .
\end{aligned}
$$

The control laws are deduced as follows supposing $\bar{F}$ is known:

$$
\begin{aligned}
u=b^{-1}[ & -k_{1}\left(z_{2}-c_{1} z_{1}\right)-f\left(x_{1}, x_{2}\right)-\bar{F} \operatorname{sgn}(\sigma) \\
& \left.+\ddot{y}_{d}-\dot{\alpha}_{1}-h(\sigma+\beta \operatorname{sgn}(\sigma))\right] .
\end{aligned}
$$

Here $h$ and $\beta$ are all positive constants.

It is not easy to obtain the boundary of external disturbances and uncertain parameters due to the highly complex space environment. The adaptive algorithm is adopted to estimate the external disturbances and uncertainties $F$ in order to retain from the boundary.

Define Lyapunov function as $V_{3}=V_{2}+(1 / 2 \gamma) \widetilde{F}^{2}$; the error is $\widetilde{F}=F^{*}-\widehat{F}$, and $\widehat{F}$ is the estimated value of $F ; \gamma$ is a positive constant. Substituting $V_{2}$ into Equation $V_{3}$ as

$$
\begin{aligned}
& \dot{V}_{3}= \dot{V}_{2}-\frac{1}{\gamma} \widetilde{F} \dot{\widehat{F}} \\
&=z_{1} z_{2}-c_{1} z_{1}^{2}+\sigma\left[k_{1}\left(z_{2}-c_{1} z_{1}\right)+f\left(x_{1}, x_{2}\right)\right. \\
&\left.+b u+F-\ddot{y}_{d}+\dot{\alpha}_{1}\right]-\frac{1}{\gamma} \widetilde{F} \dot{\widehat{F}}
\end{aligned}
$$




$$
\begin{aligned}
&=z_{1} z_{2}-c_{1} z_{1}^{2}+\sigma\left[k_{1}\left(z_{2}-c_{1} z_{1}\right)+f\left(x_{1}, x_{2}\right)\right. \\
&\left.+b u+F-\ddot{y}_{d}+\dot{\alpha}_{1}\right]-\frac{1}{\gamma} \widetilde{F}(\dot{\hat{F}}-\gamma \sigma) .
\end{aligned}
$$

The adaptive controller laws are obtained as

$$
\begin{gathered}
u=b^{-1}\left[-k_{1}\left(z_{2}-c_{1} z_{1}\right)-f\left(x_{1}, x_{2}\right)\right. \\
\left.-\widehat{F}+\ddot{y}_{d}-\dot{\alpha}_{1}-h(\sigma+\beta \operatorname{sgn}(\sigma))\right], \\
\dot{\widehat{F}}=\gamma \sigma .
\end{gathered}
$$

The stability of the controller is proved as follows. Substituting (38) into (37),

$$
\dot{V}_{3}=z_{1} z_{2}-c_{1} z_{1}^{2} h \sigma^{2}-h \beta|\sigma| .
$$

Take

$$
\begin{gathered}
Q=\left[\begin{array}{cc}
c_{1}+h k_{1}^{2} & h k_{1}-\frac{1}{2} \\
h k_{1}-\frac{1}{2} & h
\end{array}\right], \\
z^{T} Q z=\left[\begin{array}{ll}
z_{1} & z_{2}
\end{array}\right]\left[\begin{array}{cc}
c_{1}+h k_{1}^{2} & h k_{1}-\frac{1}{2} \\
h k_{1}-\frac{1}{2} & h
\end{array}\right]\left[\begin{array}{ll}
z_{1} & z_{2}
\end{array}\right]^{T} \\
=c_{1} z_{1}^{2}+h k_{1}^{2} z_{1}^{2}+2 h k_{1} z_{1} z_{2}-z_{1} z_{2}+h z_{2}^{2} \\
=c_{1} z_{1}^{2}-z_{1} z_{2}+h \sigma^{2} .
\end{gathered}
$$

Rewriting (39) to

$$
\dot{V}_{3}=z_{1} z_{2}-c_{1} z_{1}^{2}-h \sigma^{2}-h \beta|\sigma|=-z^{T} Q z-h \beta|\sigma| \leq 0
$$

$\mathrm{Q}$ is ensured to be a positive definite matrix while $h, c_{1}, k_{1}$ are appropriate values.

The desired attitude of spacecraft is adopted exponential function as

$$
z d 1=(0.1+0.15 * n * t) * \exp (-1.5 * n * t) .
$$

The higher order terms of gravitational potential are regarded as uncertainties and solar radiation pressures are regarded as disturbances which are assumed as the following equation:

$$
F\left(t, x_{1}, x_{2}\right)=\left[\begin{array}{l}
0.02 \sin (\omega t) \cdot V_{l x} \\
0.02 \sin (\omega t) \cdot V_{l y} \\
0.02 \cos (\omega t) \cdot V_{l z}
\end{array}\right]
$$

To verify and visualize the efficacy of the developed control scheme, numerical simulations under external disturbances and uncertainties are conducted using (21)-(23) and control law (38). Parameters related to operating conditions are also given about Eros 433 in Table 1.

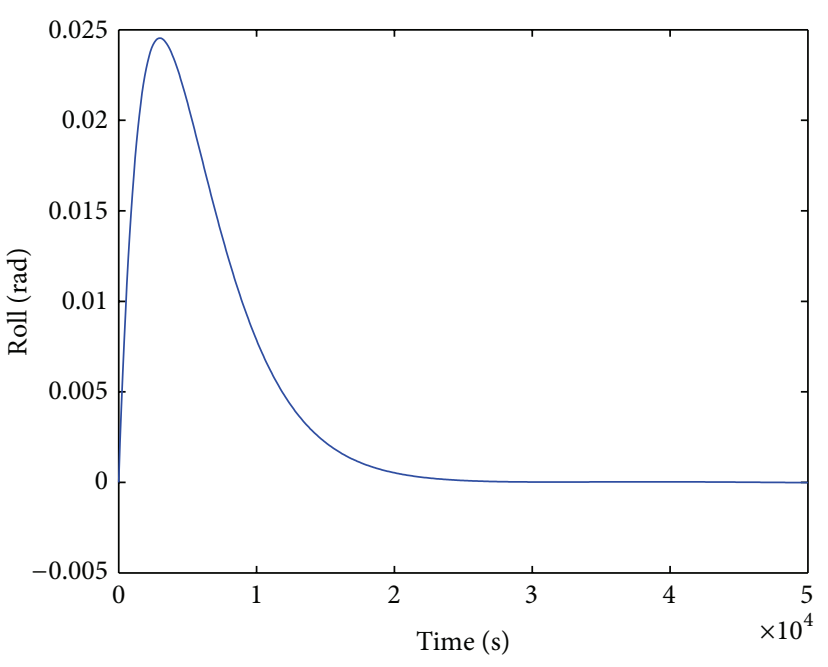

Figure 29: Roll response.

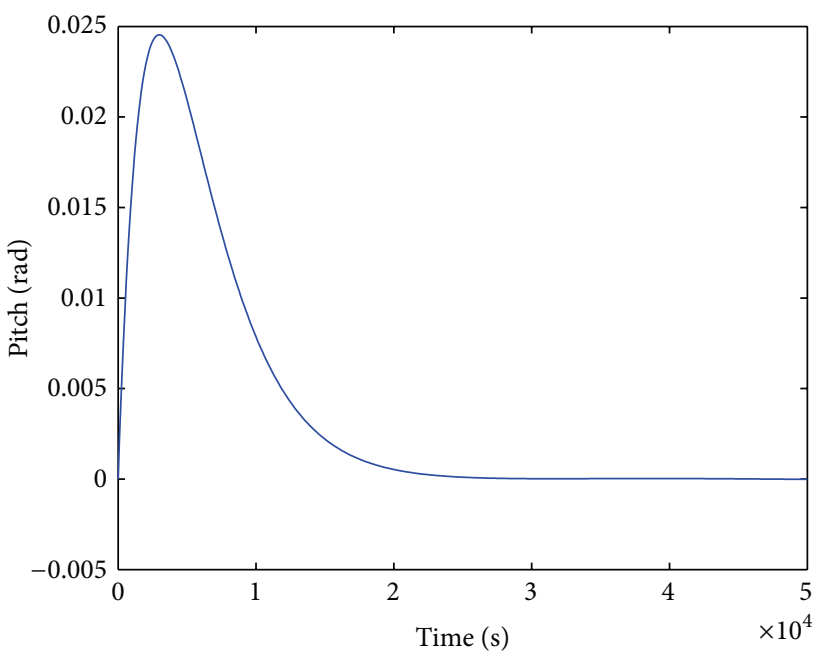

FIgURE 30: Pitch response.

Some experimental results are provided to demonstrate the effectiveness of the proposed adaptive backstepping sliding mode control laws. Figures 29, 30, and 31 give the spacecraft pitch, roll, and yaw attitude angles response motion around Eros 433. We also can obtain the pitch controller response as Figure 32. Compared with the attitude stability analysis in references [10-13], closed-loop controllers are proposed to make the spacecraft attitude angles tracking the desired attitude as (42) and reach the null state as time increases. Moreover, from simulation results one can obtain the control law neutralizing the effects of asteroid shape and mass distributions and orbital eccentricity as well as external disturbances and uncertainties described as (43). The robust control performance of the proposed adaptive backstepping sliding-mode control system is obvious than references [14, 15], which ensure stable orbiting motion and space mission. 


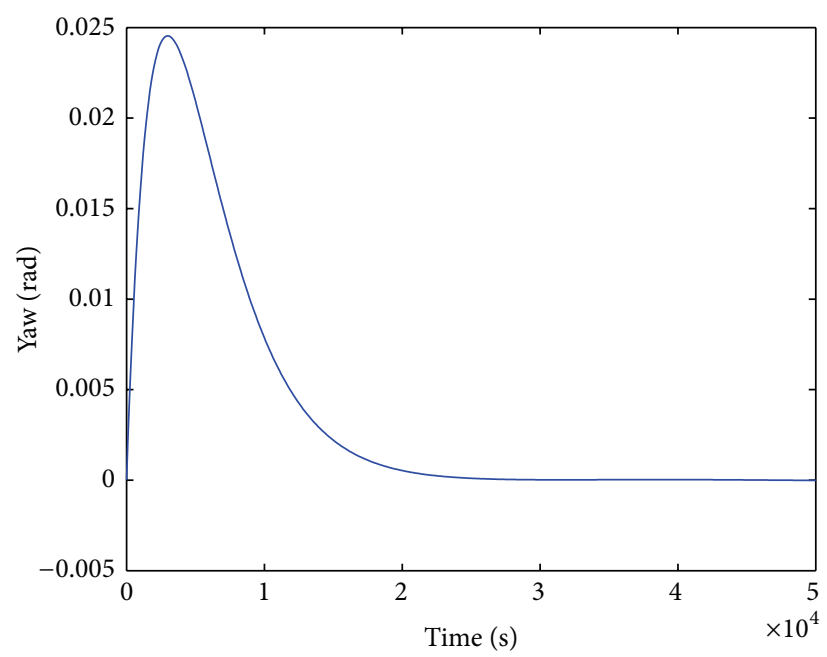

Figure 31: Yaw response.

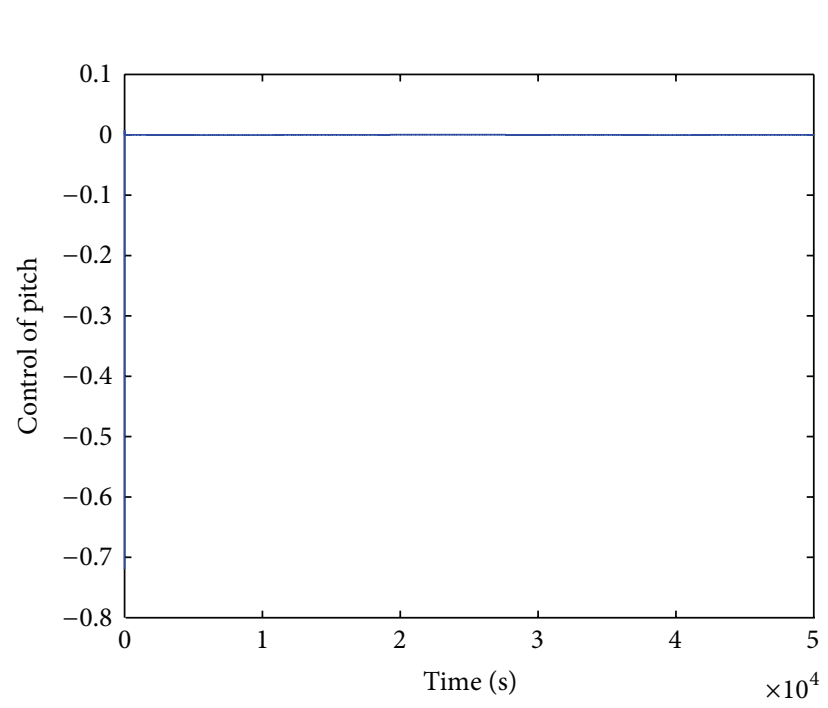

(a) Control of pitch

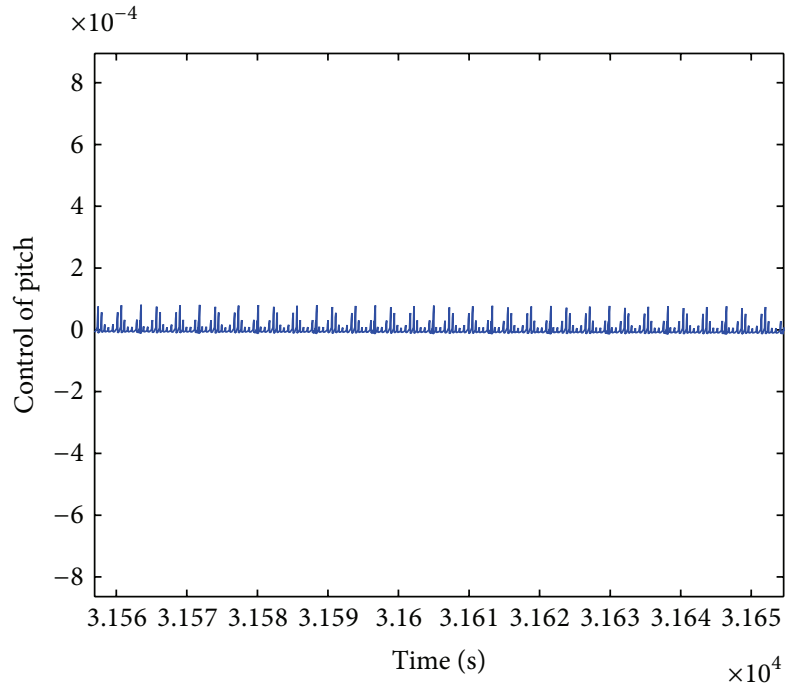

(b) Partial enlargement of control

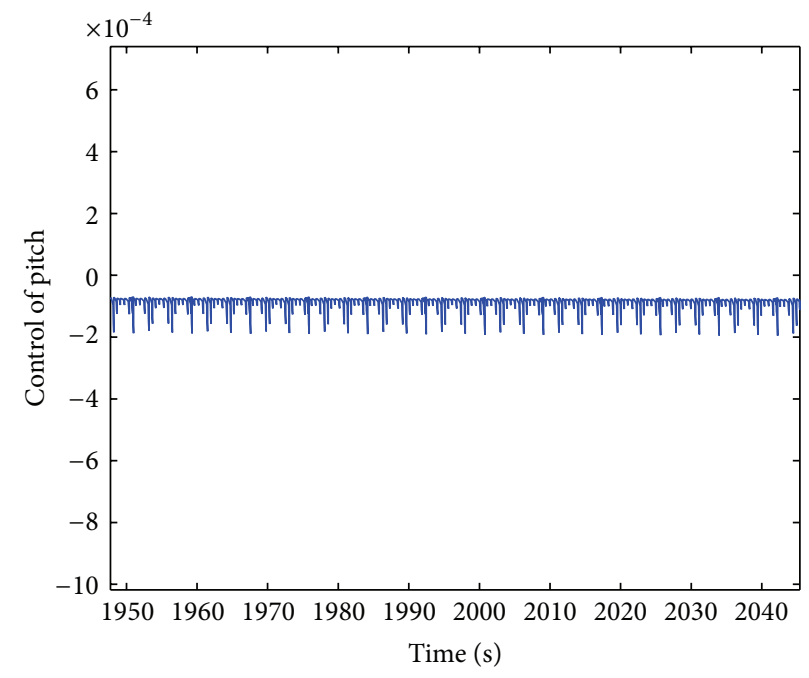

(c) Partial enlargement of control

Figure 32: Pitch control response. 


\section{Conclusions}

This paper has focused on the attitude dynamics and effect control algorithms for spacecraft orbiting rotating asteroids. Firstly, three-dimensional attitude motion of the spacecraft is examined considering the perturbation force. Then stability analysis is presented in retrograde orbits and direct orbits using linearized system model. It appears that the nonspherical shape and the rotational state of asteroids can have important effects on the attitude motion. The adaptive backstepping sliding mode control laws are designed to make the attitude angles decay and reach the null state. Computer simulations are carried out for the asteroid Eros 433 to illustrate the effectiveness of the control laws.

\section{Nomenclature}

\begin{tabular}{|c|c|}
\hline$a, b, c:$ & $\begin{array}{l}\text { Major semiaxes of the asteroid } \\
\text { considered as triaxial ellipsoid }\end{array}$ \\
\hline$(\vec{I}, \vec{J}, \vec{K}):$ & Asteroid centered inertial frame \\
\hline$(\vec{i}, \vec{j}, \vec{k}):$ & Asteroid-fixed frame \\
\hline$\left(\vec{o}_{1}, \vec{o}_{2}, \vec{o}_{3}\right):$ & Spacecraft orbital frame \\
\hline$\left(\vec{b}_{1}, \vec{b}_{2}, \vec{b}_{3}\right):$ & Spacecraft-fixed frame \\
\hline$I_{1}, I_{2}, I_{3}:$ & $\begin{array}{l}\text { Principal moments of inertia of the } \\
\text { spacecraft }\end{array}$ \\
\hline$\omega_{1}, \omega_{2}, \omega_{3}:$ & $\begin{array}{l}\text { Components of the angular velocity } \\
\text { along the principal axes in the } \\
\text { spacecraft-fixed frame }\end{array}$ \\
\hline$\omega_{b 1}, \omega_{b 2}, \omega_{b 3}:$ & $\begin{array}{l}\text { Relative angular velocity of the } \\
\text { spacecraft with respect to the orbital } \\
\text { frame }\end{array}$ \\
\hline$M_{B O}:$ & $\begin{array}{l}\text { Coordinate transformation matrix } \\
\text { from the orbital frame to the } \\
\text { spacecraft-fixed frame }\end{array}$ \\
\hline$M_{y}, M_{p}, M_{r}:$ & $\begin{array}{l}\text { Components of the external control } \\
\text { moment }\end{array}$ \\
\hline$M_{1}, M_{2}, M_{3}:$ & $\begin{array}{l}\text { Components of the gravitational field } \\
\text { of the asteroid }\end{array}$ \\
\hline$M_{\Delta 1}, M_{\Delta 2}, M_{\Delta 3}:$ & $\begin{array}{l}\text { Components of the perturbation } \\
\text { force }\end{array}$ \\
\hline$\dot{\eta}:$ & Instantaneous orbital rate \\
\hline$R_{e}:$ & Characteristic length of the primary \\
\hline$\varphi, \delta:$ & $\begin{array}{l}\text { Latitude and longitude of the orbiting } \\
\text { particle measured in an } \\
\text { asteroid-fixed frame }\end{array}$ \\
\hline$P_{l m}(\sin \varphi):$ & $\begin{array}{l}\text { Legendre polynomials of degree } l \text { and } \\
\text { order } m\end{array}$ \\
\hline$C_{l m}, S_{l m}:$ & Harmonic coefficients \\
\hline$m:$ & Spacecraft mass \\
\hline$M:$ & Asteroid mass \\
\hline$R_{c}:$ & Orbital radius \\
\hline$R:$ & $\begin{array}{l}\text { The distance of an orbiting particle } \\
\text { from the center of mass of the } \\
\text { primary }\end{array}$ \\
\hline$\lambda, \theta, \gamma:$ & Spacecraft yaw, pitch, and roll angles \\
\hline$\Omega:$ & Asteroid's spin rate \\
\hline$\rho:$ & Density of the asteroid \\
\hline$z d 1:$ & Desired attitude angle of spacecraft \\
\hline
\end{tabular}
$\mu$ : Asteroid's gravitational constant parameter.

\section{Conflict of Interests}

The authors declare that there is no conflict of interests regarding the publication of this paper.

\section{Acknowledgments}

This work is supported by National Basic Research Program of China (973 Program) under Grant 2012CB720000. The authors also gratefully acknowledge the helpful comments and suggestions of the reviewers, which have improved the presentation.

\section{References}

[1] NASA, "Planetary Science: Asteroids," http://nssdc.gsfc.nasa .gov/planetary/planets/asteroidpage.html.

[2] W. D. David, W. F. Robert, J. V. McAdams et al., "Implementation of the first asteroid landing," Icarus, vol. 159, no. 2, pp. 433-438, 2002.

[3] http://www.macgraphic.co.jp/isas/.

[4] T. Kubota, T. Hashimoto, S. Sawai et al., "An autonomous navigation and guidance system for MUSES-C asteroid landing," Acta Astronautica, vol. 52, no. 2-6, pp. 125-131, 2003.

[5] G. Schwehm and M. Hechler, “Rosetta”-ESA's planetry cornerstone mission," ESA Bulletin, no. 77, pp. 7-18, 1994.

[6] D. J. Scheeres, "Orbit mechanics about asteroids and comets," Journal of Guidance, Control and Dynamic, vol. 35, no. 3, pp. 987-997, 2012.

[7] D. J. Scheeres, "Dynamics about uniformly rotating triaxial ellipsoids: applications to asteroids," Icarus, vol. 110, no. 2, pp. 225-238, 1994.

[8] D. J. Scheeres, "Orbital mechanics about small bodies," Acta Astronautica, vol. 72, pp. 1-14, 2012.

[9] A. Rossi, F. Marzari, and P. Farinella, "Orbital evolution around irregular bodies," Earth, Planets and Space, vol. 51, no. 11, pp. 1173-1180, 1999.

[10] Y. Wang and S. Xu, "Attitude stability of a spacecraft on a stationary orbit around an asteroid subjected to gravity gradient torque," Celestial Mechanics and Dynamical Astronomy, vol. 115, no. 4, pp. 333-352, 2013.

[11] Y. Wang and $\mathrm{S}$. $\mathrm{Xu}$, "Equilibrium attitude and stability of a spacecraft on a stationary orbit around an asteroid," Acta Astronautica, vol. 84, pp. 99-108, 2013.

[12] J. L. Riverin and A. K. Misra, "Attitude dynamics of satellites orbiting small bodies," in Proceedings of the AIAA/AAS Astrodynamics Specialist Conference and Exhibit, AIAA 2002-4520, pp. 1-12, Monterey, Calif, USA, August 2002.

[13] A. K. Misra and Y. Panchenko, "Attitude dynamics of satellites orbiting an asteroid," The Journal of the Astronautical Sciences, vol. 54, no. 3-4, pp. 369-381, 2006.

[14] K. D. Kumar and M. Shah, "Attitude dynamics and control of satellites orbiting rotating asteroids," in Proceedings of the Canadian Conference on Electrical and Computer Engineering (CCECD '07), pp. 1388-1391, Vancouver, Canada, April 2007. 
[15] R. Mahmut, N. K. Niloofar, and T. Kuhara, "Orbital and attitude control of a spacecraft around an asteroid," in Proceedings of the 12th International Conference on Control, Automation and Systems (ICCAS '12), pp. 1627-1632, JeJu Island, Republic of Korea, October 2012.

[16] L. Wu, W. X. Zheng, and H. Gao, "Dissipativity-based sliding mode control of switched stochastic systems," IEEE Transactions on Automatic Control, vol. 58, no. 3, pp. 785-791, 2013.

[17] L. Wu, X. Su, and P. Shi, "Sliding mode control with bounded $\mathscr{L}_{2}$ gain performance of Markovian jump singular time-delay systems," Automatica, vol. 48, no. 8, pp. 1929-1933, 2012.

[18] Y. Song, X. Li, and W. Cai, "Adaptive and fault-tolerant reactive power compensation in power systems via multilevel STATCOMs," International Journal of Innovative Computing, Information and Control, vol. 9, no. 8, pp. 3403-3413, 2013.

[19] H. Zhang, Y. Shi, and B. Mu, "Optimal $\mathscr{H}_{\infty}$-based linear-quadratic regulator tracking control for discrete-time Takagi-Sugeno fuzzy systems with preview actions," Journal of Dynamic Systems, Measurement, and Control, vol. 135, no. 4, Article ID 044501, 5 pages, 2013.

[20] Z. Shuai, H. Zhang, J. Wang, J. Li, and M. Ouyang, "Combined AFS and DYC control of four-wheel-independent-drive electric vehicles over CAN network with time-varying delays," IEEE Transactions on Vehicular Technology, vol. 63, no. 2, pp. 591-602, 2014.

[21] C. Stefano and M. Susanna, "Methods for computing the potential of an irregular, homogeneous, solid body and its gradient," in Proceedings of the AIAA/AAS Astrodynamics Specialist Conference, AOO-39767, AIAA 2002-4023, pp. 82-96, Monterey, Calif, USA, August 2002.

[22] W. Boyce, "Comment on a formula for the gravitational harmonic coefficients of a triaxial ellipsoid," Celestial Mechanics \& Dynamical Astronomy, vol. 67, no. 2, pp. 107-110, 1997.

[23] A. J. Koshkouei and K. J. Burnham, "Adaptive backstepping sliding mode control for feedforward uncertain systems," International Journal of Systems Science, vol. 42, no. 12, pp. 1935-1946, 2011. 


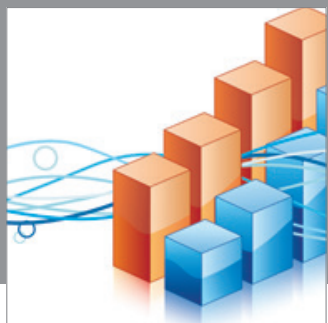

Advances in

Operations Research

mansans

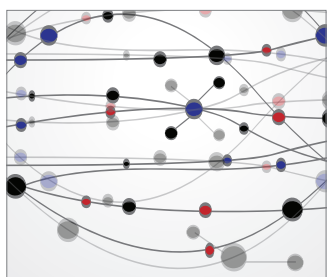

The Scientific World Journal
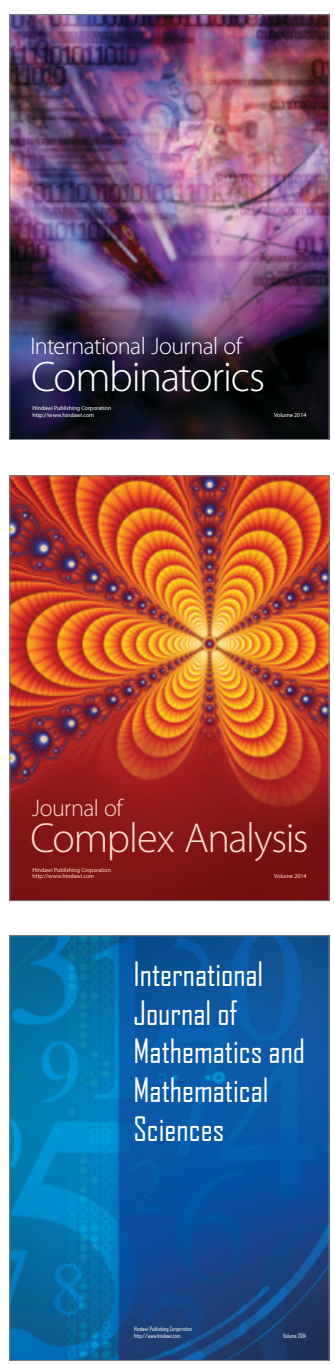
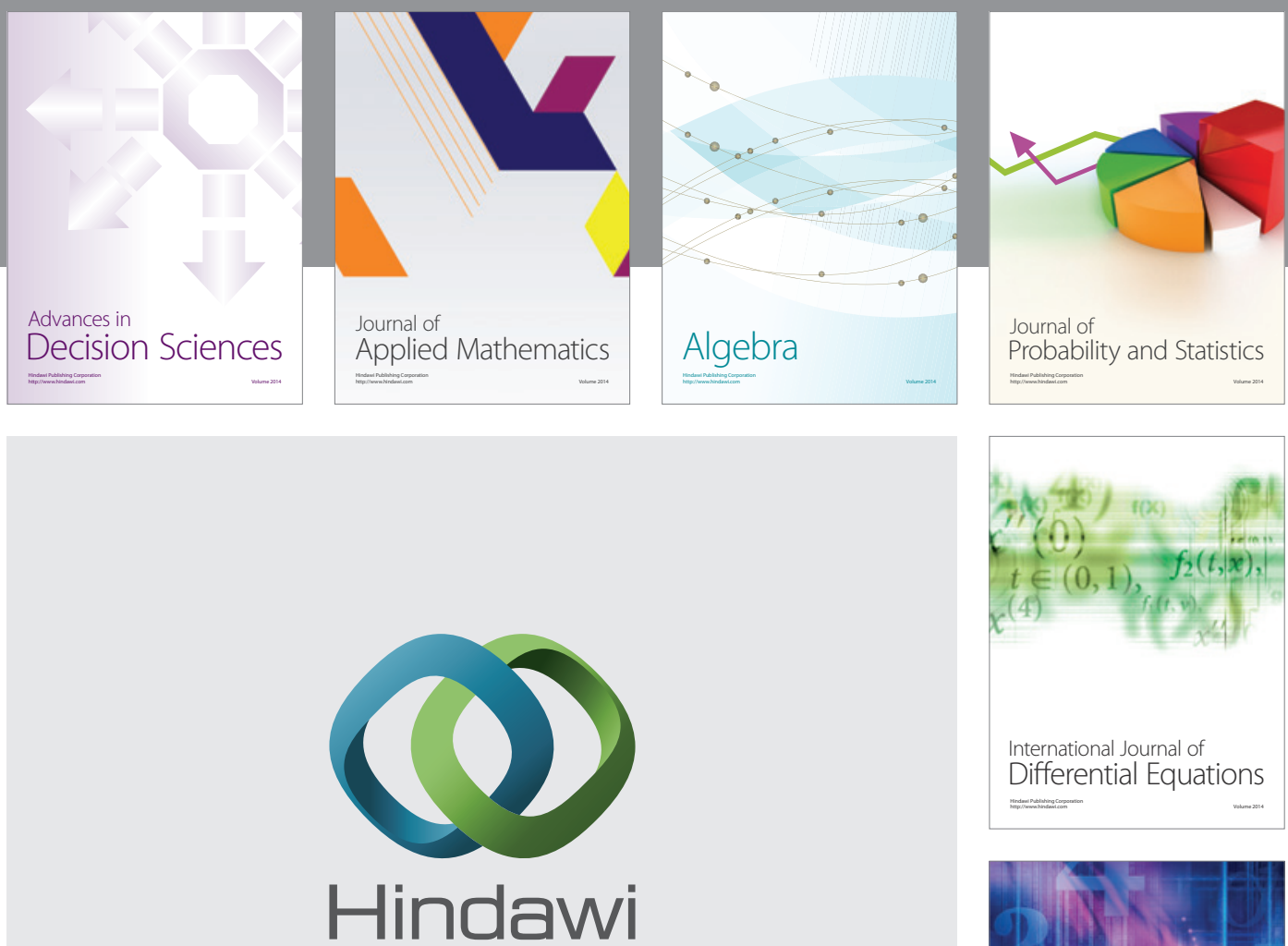

Submit your manuscripts at http://www.hindawi.com
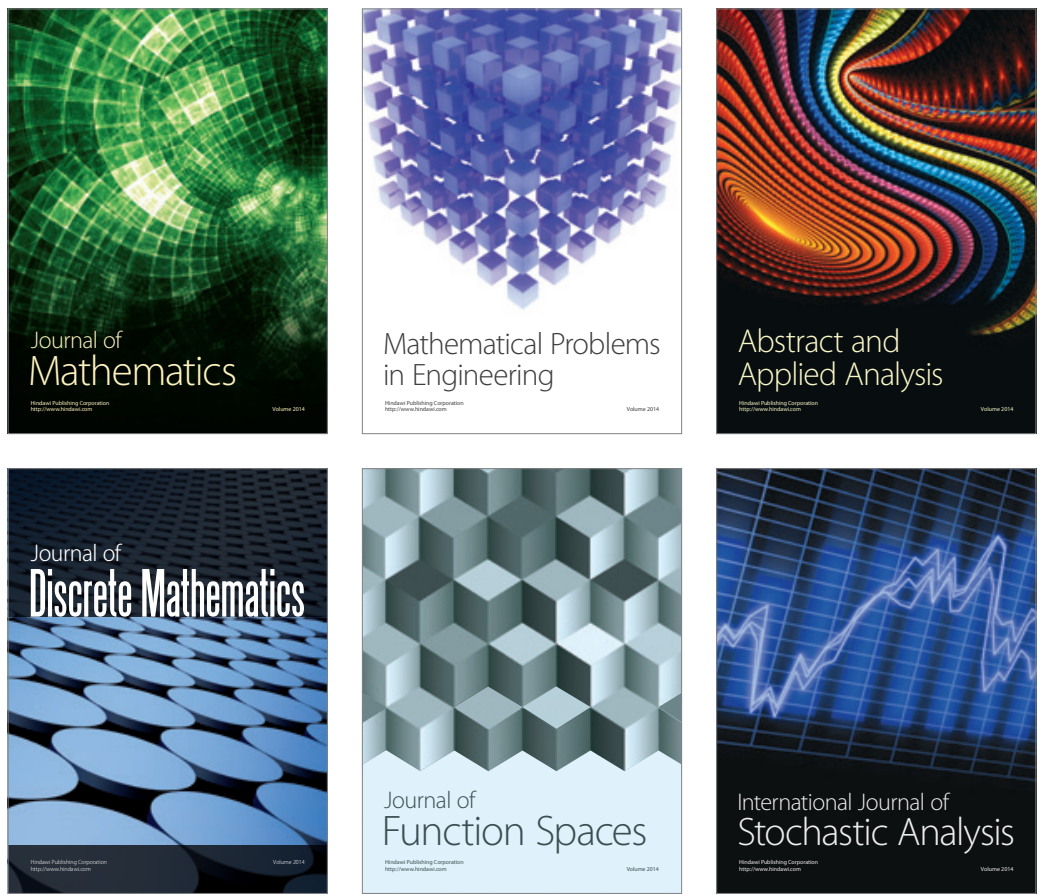

Journal of

Function Spaces

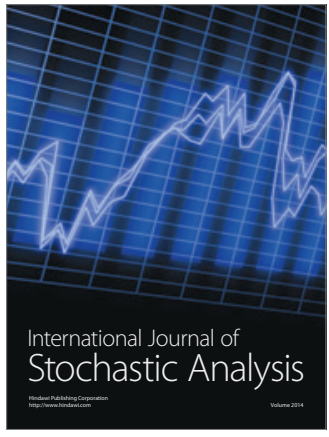

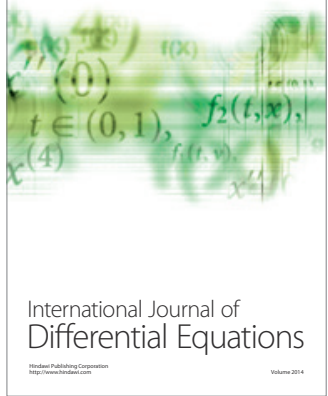
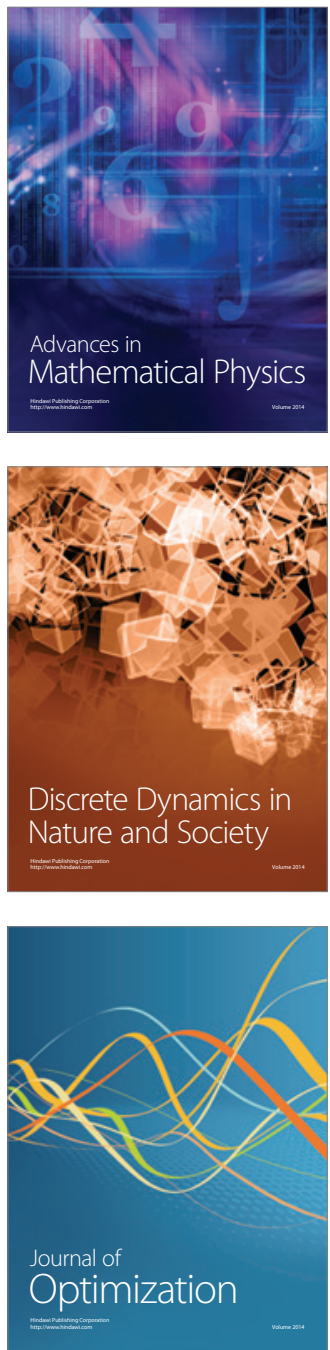